\title{
Time scale separation in the vector borne disease model SIRUV via center manifold analysis
}

\author{
Maíra Aguiar ${ }^{1,2,3}$, Bob Kooi ${ }^{4}$, Andrea Pugliese ${ }^{2}$, \\ Mattia Sensi ${ }^{2} \&$ Nico Stollenwerk ${ }^{1,2,5 \#}$ \\ ${ }^{1}$ Basque Center for Applied Mathematics (BCAM), \\ Alameda Mazarredo 14, 48009 Bilbao, Spain, \\ ${ }^{2}$ Dipartimento di Matematica, Università degli Studi di Trento, \\ Via Sommarive 14, 38123 Povo (Trento), Italy, \\ ${ }^{3}$ Ikerbasque, Basque Foundation for Science, Bilbao, Spain, \\ ${ }^{4}$ Vrije Universiteit Amsterdam, de Boelelaan, Amsterdam, The Netherlands, \\ ${ }^{5}$ CMAF-CIO, Lisbon University, Campo Grande, Lisbon, Portugal, \\ \# corresponding author's e-mail address: nico.biomath@gmail.com
}

April 6, 2021

\begin{abstract}
We investigate time scale separation in the vector borne disease model SIRUV, as previously described in the literature [1], and recently reanalyzed with the singular perturbation technique [2]. We focus on the analysis with a single small parameter, the birth and death rate $\mu$, whereas all other model parameters are much larger and describe fast transitions. The scaling of the endemic stationary state, the Jacobian matrix around it and its eigenvalues with this small parameter $\mu$ is calculated and the center manifold analysis performed with the method described in [3] which goes back to earlier work $[4,5]$, namely a transformation of the Jacobian matrix to block structure in zeroth order in the parameter $\mu$ is used and then a family of center manifolds with $\mu$ larger than zero is obtained.
\end{abstract}

\section{Introduction}

Here we investigate the time scale separation of the fast infected mosquito dynamics $V$ from the slow human infection $I$ in the SIRUV model in respect to its only small parameter $\mu$, describing the slow transition of building up susceptibles $S$ from the recovered $R$, either by waning immunity or here via death of any human and birth of susceptibles.

The SIRUV model has been described in detail in [1] and recently investigated further e.g. in [2]. However, the full SIRUV model does not have the standard form for time scale separation, where the standard form is a separation into 
slow variable dynamics $d x / d t=\varepsilon f(x, y, \varepsilon)$ and fast dynamics $d y / d t=g(x, y, \varepsilon)$ with a small scaling parameter $\varepsilon$, eventually originating from scaling of several biological model parameters. In [1] from the SIRUV a simplified model, the SISUV model, was constructed by using nearly all parameters as in the SIRUV model, but without a recovered class $R$, and therefore an extended period of infection, hence slow recovery, i.e. small $\gamma$, and consequently also small infectivity $\beta$. Hence the small parameter $\varepsilon$ originates from $\gamma=\varepsilon \cdot \bar{\gamma}$ and $\beta=\varepsilon \cdot \bar{\beta}$ with $\bar{\beta}$ etc. in the range of the fast mosquito parameters. This simplified SISUV model has the standard form for time scale separation, and hence is a good test bed for standard techniques in comparison, such as center manifold analysis in comparison with classical time scale separation arguments like scaling of the time parameter with $\varepsilon$ to obtain quasistationary expressions $V=V(I)$, all this in zeroth order implicitly [1] or more recently a rigorous analysis with singular perturbation techniques beyond the zeroth order approximation [2].

But in the full SIRUV model we only have one biologically small parameter $\mu$ inside the slow dynamics function $f=f(x, y, \mu)$ and hence slow dynamics $d x / d t=f(x, y, \mu)$ and fast dynamics $d y / d t=g(x, y)$. For such harder problems of time scale separation in the literature other techniques like Implicit Low Dimensional Manifolds (ILDM) or zero-derivative principle for slow-fast dynamical systems have been suggested and compared with each other [6]. The ILDM e.g. relies on the Jacobian matrix around any point in state space and the condition for slow manifold is given by the expression of the dynamics in eigenvector basis.

In standard time scale separation systems the comparison of ILDM and singular perturbation have shown that they agree only in lower order approximation up to second order $\left(\mathcal{O}\left(\varepsilon^{2}\right)\right)$, but then deviate [7]. And such methodes like ILDM or zero-derivative principle are in danger of picking up spurious manifolds not agreeing with the Fenichel manifold of singular perturbation, in cases when they can be compared [6].

Here the center maifold analysis for the SIRUV model is presented as closer to the singular perturbation theory (and actually in a careful analysis of the scaling with small parameter the center manifold and singular perturbation agree in standard time scale separation systems, see the SISUV model again as example). Actually, a singular perturbation analysis can be performed in the SIRUV model by introducing next to the naturally small parameter $\mu$ a second parameter $\varepsilon$ in the way of $d x / d t=\varepsilon f(x, y, \mu)$ and $d y / d t=g(x, y)$ where $\varepsilon$ is not directly originating from the model parameters, but based on dimensionality arguments originating from the eigenvalue structure of the Jacobian matrix around the endemic fixed point [2]. Then $\varepsilon$ has to be considered as small in respect to the mortality of the mosquitoes $\nu$, compared to the mortality of the humans $\mu$, but is not given directly from the model parameters and the dimensionality question has to be dealt with via extra arguments. However, the singular perturabtion gives fast calculations of high orders of the Fenichel manifold, and is therefore attractive for quick analytical treatment.

Here, in the center manifold analysis a second small parameter is not needed, and a series expansion can be obtained for the naturally small parameter $\mu$ and for the slow state space variables $x$ giving the fast variable as manifold 
$y=h(x, \mu)=\sum_{\nu=1}^{\infty} \mu^{\nu} \sum_{\omega=1}^{\infty} h_{\nu, \omega} x^{\omega}$, where $x$ can be higher dimensional. The number of slow versus fast variables in all these methods is determined by a spectral gap, i.e. eigenvalues with small real part for the slow variables and such with much larger real part for the fast contraction directions in state space, see e.g. [6].

Another scaling has been suggested, based on the stationary states $S^{*}, I^{*}$ and $V^{*}$, i.e. $I=\mu \bar{I}$ and $V=\mu \bar{V}$ with $\bar{I}, \bar{V} \approx S^{*}$ (Trento scaling), instead of the scaling from the spectral gap in the eigenvalues of the Jacobian matrix around the endemic stationary state. This scaling of state variables, however, describes a slow-fast dynamics of slow susceptibles $S$ and fast infected humans $I$ and infected mosquitoes $V$ in the transient behaviour, before the system finally enters into the slow two-dimensional subspace of $V=V(S, I)$ with $S$ and $I$ slow and only $V$ fast, as observed from the scaling of the eigenvalues around the endemic stationary state.

\section{The SIRUV model revisited}

The SIRUV model (see [1] for a detailed description) reads as follows

$$
\begin{aligned}
\frac{d}{d t} S & =\mu(N-S)-\frac{\beta}{M} S V \\
\frac{d}{d t} I & =\frac{\beta}{M} S V-(\gamma+\mu) I \\
\frac{d}{d t} R & =\gamma I-\mu R \\
\frac{d}{d t} U & =\psi-\nu U-\frac{\vartheta}{N} U I \\
\frac{d}{d t} V & =\frac{\vartheta}{N} U I-\nu V
\end{aligned}
$$

with human population size $N=S(t)+I(t)+R(t)$ and mosquito population size $M=U(t)+V(t)$ assumed constant, hence for now $\psi:=\nu \cdot M$. In cosiderations of seasonality of mosquito abundance $\psi$ could become seasonally forced [11].

For the human population we have birth and death rate of $\mu=\frac{1}{65 y}$, recovery rate for e.g. dengue fever of around $\gamma=\frac{1}{7 d}=\frac{365}{7} \mathrm{y}^{-1}$, and infection rate $\beta=2 \cdot \gamma$. And for the mosquitoes life expectancy of adult mosquitoes, since only female mosquitoes bite humans for their egg production, we have $\nu=$ $\frac{1}{10 \mathrm{~d}}=\frac{365}{10} \mathrm{y}^{-1}$, birth rate for a stable population $\psi=\nu \cdot M$ and infection rate $\vartheta=2 \cdot \nu[1]$.

We observe that we have only one slow parameter, the human life span or supply of new susceptibles, $\mu=\frac{1}{65 \mathrm{y}}=0.01538 \mathrm{y}^{-1}$. All other parameters are fast, since we have $\gamma=\frac{1}{7 \mathrm{~d}}=\frac{365}{7} \mathrm{y}^{-1}=52.14 \mathrm{y}^{-1}, \beta=2 \cdot \gamma=104.29 \mathrm{y}^{-1}$, $\nu=\frac{1}{10 \mathrm{~d}}=\frac{365}{10} \mathrm{y}^{-1}=36.5 \mathrm{y}^{-1}$ and $\vartheta=2 \cdot \nu=73.0 \mathrm{y}^{-1}$. For any numerical analysis we might use as population sizes $N=10^{6}$ and $M=10 \cdot N$, hence the ratio of mosquitos to humans is $\kappa=10$.

Due to the conservation of population sizes we can reduce the SIRUV model 
to e.g. an SIV model given by

$$
\begin{aligned}
\frac{d}{d t} S & =\mu(N-S)-\frac{\beta}{M} S V \\
\frac{d}{d t} I & =\frac{\beta}{M} S V-(\gamma+\mu) I \\
\frac{d}{d t} V & =\frac{\vartheta}{N}(M-V) I-\nu V
\end{aligned}
$$

as considered recently [2]. Previously, we investigated an SRV version of the SIRUV model, since $S$ and $R$ both are in stationarity macroscopic variables, whereas $V$ is of order $\mu$, hence small and decreasing rapidly with decreasing $\mu$ [1]. But the biology, of course, does not change with the variables chosen for the analysis.

\subsection{The endemic stationary state of the SIRUV model}

The endemic stationary state of the SIRUV model is given by

$$
V^{*}=\frac{\frac{\vartheta}{\nu} I^{*}}{N+\frac{\vartheta}{\nu} I^{*}} \cdot M
$$

for the infected mosquitoes $V$, and

$$
I^{*}=\frac{\mu}{\gamma+\mu}\left(1-\frac{\gamma+\mu}{\frac{\vartheta}{\nu} \beta}\right) \frac{\beta}{\beta+\mu} \cdot N
$$

for the infected humans $I$, and

$$
S^{*}=N-\frac{\gamma+\mu}{\mu} I^{*}=\left(\frac{\gamma+\mu}{\frac{\vartheta}{\nu} \beta}+\frac{\mu}{\beta+\mu}\right) \cdot N
$$

for the susceptible humans. The other variables $R$ and $U$ follow from the conservations, hence $R^{*}=N-S^{*}-I^{*}$ and $U^{*}=M-V^{*}$.

Of special interest for the following is the fact that $I^{*}$ and with it also $V^{*}$ scale with the small parameter $\mu$, hence are of order $\mathcal{O}(\mu)$, whereas $S^{*}$ is of order $\mathcal{O}(1)$, see Eqs. (88), (89) and (90) below.

\subsection{Stability analysis around the endemic stationary state of the SIRUV model}

With the notation

$$
\begin{aligned}
\frac{d}{d t} S & =\mu(N-S)-\frac{\beta}{M} S V=: f(S, I, V) \\
\frac{d}{d t} I & =\frac{\beta}{M} S V-(\gamma+\mu) I=: g(S, I, V) \\
\frac{d}{d t} V & =\frac{\vartheta}{N}(M-V) I-\nu V=: h(S, I, V)
\end{aligned}
$$


we calculate the Jacobian matrix of the SIRUV model around the endemic stationary state

$$
\begin{aligned}
A= & \left.\left(\begin{array}{lll}
\frac{\partial f(S, I, V)}{\partial S}\left(\frac{\partial f(S, I, V)}{\partial I}\right. & \frac{\partial f(S, I, V)}{\partial V} \\
\frac{\partial g(S, I, V)}{\partial S} & \left.\frac{\partial g(S, I, V)}{\partial I}\right) & \frac{\partial g(S, I, V)}{\partial V} \\
\frac{\partial h(S, I, V)}{\partial S} & \frac{\partial h(S, I, V)}{\partial I} & \frac{\partial h(S, I, V)}{\partial V}
\end{array}\right)\right|_{\left(S^{*}, I^{*}, V^{*}\right)} \\
= & \left(\begin{array}{ccc}
-\frac{\beta}{M} \cdot V^{*}-\mu & 0 & -\frac{\beta}{M} \cdot S^{*} \\
\frac{\beta}{M} \cdot V^{*} & -(\gamma+\mu) & \frac{\beta}{M} \cdot S^{*} \\
0 & \frac{\vartheta}{N} \cdot\left(M-V^{*}\right) & -\frac{\vartheta}{N} I^{*}-\nu
\end{array}\right)=:\left(\begin{array}{lll}
a_{11} & a_{12} & a_{13} \\
a_{21} & a_{22} & a_{23} \\
a_{31} & a_{32} & a_{33}
\end{array}\right)
\end{aligned}
$$

and calculate the eigenvalues of $A$. The characteristic polynomial to calculate the eigenvalues is given by

$$
0=\left(\lambda-a_{11}\right)\left(\lambda-a_{22}\right)\left(\lambda-a_{33}\right)-(\lambda+\mu) a_{23} a_{32}
$$

due to the structure of the Jacobian matrix $A$ with e.g. $a_{13}=-a_{23}$ and $a_{11}=-a_{21}-\mu$. Multiplying out we obtain the form

$$
0=\lambda^{3}+a_{2} \lambda^{2}+a_{1} \lambda+a_{0}
$$

with

$$
\begin{aligned}
a_{2}= & -\left(a_{11}+a_{22}+a_{33}\right) \\
= & \gamma+\nu+\frac{\vartheta}{N} I^{*}+\frac{\beta}{M} \cdot V^{*}+2 \mu \\
a_{1}= & a_{11} a_{22}+a_{33}\left(a_{11}+a_{22}\right)-a_{23} a_{32} \\
= & (\gamma+\mu)\left(\frac{\beta}{M} \cdot V^{*}+\mu\right)+\left(\nu+\frac{\vartheta}{N} I^{*}\right)\left(\gamma+\frac{\beta}{M} \cdot V^{*}+2 \mu\right) \\
& -\vartheta \beta \frac{S^{*}}{N}\left(1-\frac{V^{*}}{M}\right) \\
a_{0}= & -a_{11} a_{22} a_{33}-\mu a_{23} a_{32} \\
= & (\gamma+\mu)\left(\nu+\frac{\vartheta}{N} I^{*}\right)\left(\frac{\beta}{M} \cdot V^{*}+\mu\right)
\end{aligned}
$$

Now we can solve the characteristic polynomial of third order in $\lambda$ with Cardano's method, see Appendix A for the detailed analysis.

We expect a structure of the eigenvalues as follows: We have two complex eigenvalues $\lambda_{1 / 2}=a \pm i \cdot b$ for the rotating part of the trajectory into the fixed point and one real eigenvalue $\lambda_{3}=c$, with $a, b$ and $c$ real numbers [1].

From Cardano's method we obtain the eigenvalues numerically, see Appendix A, as

$$
\lambda_{1 / 2}=a \pm i \cdot b=-0.029909 \pm i \cdot 0.994780
$$

and

$$
\lambda_{3}=c=-88.67601
$$


in good numerical agreement with [1], where the SRV submodel was used, and not as here the SIV submodel.

And the scaling analysis gives the largely negative real eigenvalue as

$$
\lambda_{3}=c=-(\gamma+\nu)-\left(1+\frac{\vartheta}{\gamma}-\frac{\nu}{\beta}-\frac{\vartheta-\nu \frac{\gamma}{\beta}}{\gamma+\nu}+\frac{\vartheta \beta-\nu \gamma}{(\gamma+\nu)^{2}}\right) \cdot \mu+\mathcal{O}\left(\mu^{3 / 2}\right)
$$

with an order $\mathcal{O}(1)$ leading part and the pair of complex conjugate eigenvalues $\lambda_{1 / 2}=a \pm i b$ with real part $a$ of order $\mathcal{O}(\mu)$ and complex part $b$ of order $\mathcal{O}\left(\mu^{1 / 2}\right)$ explicitly as

$$
a=-\frac{1}{2}\left(\frac{\vartheta \beta}{\nu \gamma}+\frac{\vartheta-\nu \frac{\gamma}{\beta}}{\gamma+\nu}-\frac{\vartheta \beta-\nu \gamma}{(\gamma+\nu)^{2}}\right) \cdot \mu+\mathcal{O}\left(\mu^{3 / 2}\right)
$$

and

$$
b=\sqrt{\frac{\vartheta \beta-\nu \gamma}{\gamma+\nu}} \cdot \sqrt{\mu}+\mathcal{O}\left(\mu^{3 / 2}\right)
$$

with the detailed calculations given in Appendix A.

\subsection{Qualitative behaviour of the SIRUV model near the en- demic stationary state}

Since we analyse the long term dynamics into the endemic fixed point, we show here trajectories with initial conditions close to the fixed point. In Fig. 1 a) we observe in the time series of infected humans $I$ oscillations into the fixed point given by the straight line. The state space plot of infected humans $I$ versus susceptible humans $S$ in Fig. 1 b) shows after a brief transient parallel to the y-axis, hence fast decreasing numbers of infected the spiraling into the fixed point with little non-linear deformation left.

a)

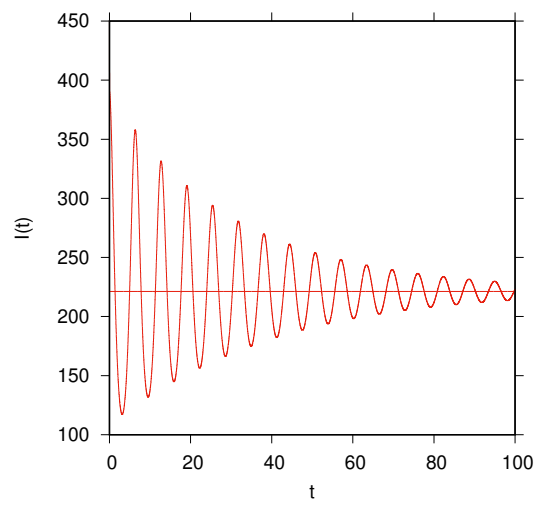

b)

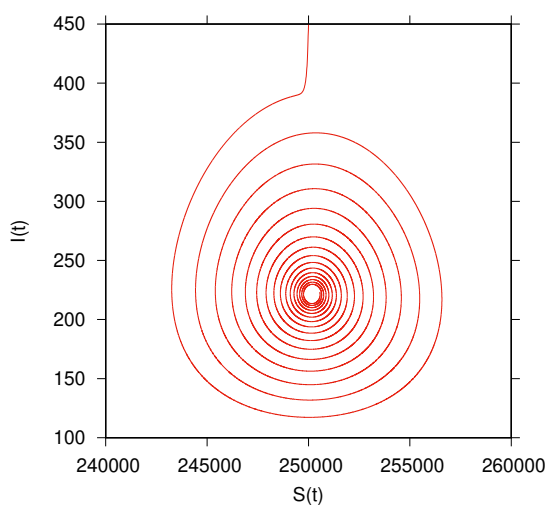

Figure 1: a) Time series of infected and b) state space plot of infected versus susceptibles in the SIRUV model with initial conditions close to the endemic fixed point. After an initial transient of decreasing infected I, oscillations symmetrically around the fixed point are observed.

For the infected mosquitoes $V$ the time series in Fig. 2 a) shows a similar dynamics of oscillations into the fixed point as the human infected $I$ in Fig. 1 
a). In addition the state space plot in Fig. 2 b) reveals, after a brief transient, a close to linear functional relation between $V$ and $I$, namely

$$
V(I) \approx \frac{\vartheta}{\nu} \cdot \frac{M}{N} \cdot I
$$

and with the parameter values used here for the SIRUV model, we obtain in good approximation $V(I) \approx 2 \cdot 10 \cdot I(t)$. There is only a minor tilting visible to just observe a spiraling in the complete three dimensional state space of $S, I$ and $V$ from this two dimensional projection of $I$ and $V$ only.

a)

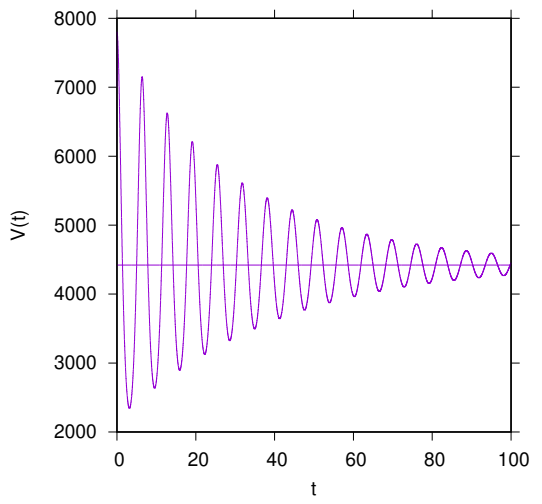

Figure 2: a) Time series of infected mosquitoes and b) state space plot of infected humans versus infected mosquitoes in the SIRUV model with initial conditions close to the endemic fixed point. The infected mosquitoes follow after a short transient period the infected humans with $V(I) \approx \frac{\vartheta}{\nu} \cdot \frac{M}{N} \cdot I=2 \cdot 10 \cdot I(t)$.

For more information in larger regions of the state space see graphs in [2], as well as here in SIV state space formulation, and similarly for the SRV state space formulation of the SIRUV model in [1]. 


\section{Center manifold analysis of the SIRUV model}

\subsection{General ansatz of the center manifold analysis of the SIRUV}

For the further analysis of the center manifold we first transform the dynamical system so that the endemic fixed point is in the center of the coordinate system.

The original SIRUV system is given by

$$
\begin{aligned}
\frac{d}{d t} S & =\mu(N-S)-\frac{\beta}{M} S V=: f_{1}(S, I, V) \\
\frac{d}{d t} I & =\frac{\beta}{M} S V-(\gamma+\mu) I=: f_{2}(S, I, V) \\
\frac{d}{d t} V & =\frac{\vartheta}{N}(M-V) I-\nu V=: f_{3}(S, I, V)
\end{aligned}
$$

and with centered coordinates

$$
\underline{z}=\left(\begin{array}{c}
z_{1} \\
z_{2} \\
z_{3}
\end{array}\right):=\left(\begin{array}{c}
S(t)-S^{*} \\
I(t)-I^{*} \\
V(t)-V^{*}
\end{array}\right)
$$

and hence for the original state variables

$$
\left(\begin{array}{c}
S \\
I \\
V
\end{array}\right)=\left(\begin{array}{c}
z_{1}+S^{*} \\
z_{2}+I^{*} \\
z_{3}+V^{*}
\end{array}\right)
$$

we obtain

$$
\begin{aligned}
\frac{d}{d t} \underline{z} & =\frac{d}{d t}\left(\begin{array}{c}
S \\
I \\
V
\end{array}\right)=\left(\begin{array}{c}
f_{1}(S, I, V) \\
f_{2}(S, I, V) \\
f_{3}(S, I, V)
\end{array}\right) \\
& =\left(\begin{array}{c}
\mu\left(N-\left(z_{1}+S^{*}\right)\right)-\frac{\beta}{M}\left(z_{1}+S^{*}\right)\left(z_{3}+V^{*}\right) \\
\frac{\beta}{M}\left(z_{1}+S^{*}\right)\left(z_{3}+V^{*}\right)-(\gamma+\mu)\left(z_{2}+I^{*}\right) \\
\frac{\vartheta}{N}\left(M-\left(z_{3}+V^{*}\right)\right)\left(z_{2}+I^{*}\right)-\nu\left(z_{3}+V^{*}\right)
\end{array}\right) \\
& =A \underline{z}+\left(\begin{array}{c}
-\frac{\beta}{M} z_{1} z_{3} \\
\frac{\beta}{M} z_{1} z_{3} \\
-\frac{\vartheta}{N} z_{3} z_{2}
\end{array}\right)=A \underline{z}+\underline{q}(\underline{z})
\end{aligned}
$$

with $A$ the Jacobian matrix of the SIRUV model around the endemic stationary state and $\underline{q}(\underline{z})$ the nonlinear part of the dynamics. Hence we have in centered coordinates the system given by

$$
\frac{d}{d t} \underline{z}=A \underline{z}+\underline{q}(\underline{z})
$$

with the non-linear parts calculated as

$$
\left(\begin{array}{l}
q_{1}(\underline{z}) \\
q_{2}(\underline{z})
\end{array}\right)=\left(\begin{array}{c}
-1 \\
1
\end{array}\right) \frac{\beta}{M} z_{1} z_{3}
$$


and

$$
q_{3}(\underline{z})=-\frac{\vartheta}{N} z_{3} z_{2}
$$

Then with the knowledge of the eigenvalues and its scaling we could calculate the, in this case, complex eigenvectors $\underline{u}_{i}$ and with the transformation matrix

$$
T=\left(\underline{u}_{1}, \underline{u}_{2}, \underline{u}_{3}\right)
$$

and the transformation $\underline{z}=T \underline{x}_{3}$ we obtain the diagonalized system with eigenvalue matrix $\Lambda$ in the form

$$
\frac{d}{d t} \underline{x}_{3}=T^{-1} A T \underline{x}_{3}+T^{-1} \underline{q}\left(T \underline{x}_{3}\right)=\Lambda \underline{x}_{3}+T^{-1} \underline{q}\left(T \underline{x}_{3}\right)
$$

The components of the in this case 3 -dimensional vector $\underline{x}_{3}$ are in time scale analysis typically grouped as

$$
\underline{x}_{3}=\left(\begin{array}{c}
x_{1} \\
x_{2} \\
y
\end{array}\right)
$$

since the first two eigenvalues in $\Lambda, \lambda_{1 / 2}=a \pm i b$, are small, corresponding to the state variables $\underline{x}:=\left(x_{1}, x_{2}\right)^{\text {tr }}$ in the transformed diagonalized system as slow variables, and the third eigenvalue is largely negative, $\lambda_{3}=c$, corresponding to the state variables $y$ in the transformed diagonalized system as fast variable.

Hence we then have the system separated into fast and slow variables given as

$$
\begin{aligned}
\frac{d}{d t} \underline{x} & =A_{2} \underline{x}+\underline{f}_{n l}(\underline{x}, y) \\
\frac{d}{d t} y & =\lambda_{3} y+g_{n l}(\underline{x}, y)
\end{aligned}
$$

because of the spectral gap between the small $\lambda_{1 / 2}=a \pm i b$ and the largely negative $\lambda_{3}=c$. The nonlinear parts $\underline{f}_{n l}(\underline{x}, y)$ and $g_{n l}(\underline{x}, y)$ have to be determined from $T^{-1} \underline{q}\left(T \underline{x}_{3}\right)$.

Here in place of $A_{2}$ with index for a 2 dimensional matrix, we could simply set the matrix of the two eigenvalues

$$
\Lambda_{2}=\left(\begin{array}{cc}
\lambda_{1} & 0 \\
0 & \lambda_{2}
\end{array}\right)=\left(\begin{array}{cc}
a+i b & 0 \\
0 & a-i b
\end{array}\right)
$$

or, instead of using complex eigenvectors, we can set a real matrix, which has the same eigenvalues as $\Lambda_{2}$, e.g. as

$$
A_{2}=\left(\begin{array}{cc}
a & b \\
-b & a
\end{array}\right)
$$

Then the full 3 dimensional matrix $A_{3}$ in block structure is given by

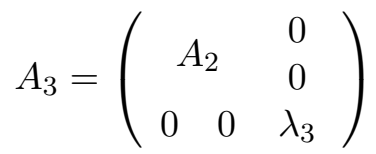


Then the center manifold is given by the fast variable $y$ as a function of the slow variables $\underline{x}:=\left(x_{1}, x_{2}\right)^{\operatorname{tr}}$

$$
y=h(\underline{x})=h\left(x_{1}, x_{2}\right)
$$

where $h$ is now in the SIRUV model a vector valued function and the functional equation $\mathcal{N}(h(\underline{x}))=0$ becomes

$$
0=\mathcal{N}(h(\underline{x}))=\frac{d h(\underline{x})}{d \underline{x}} \cdot\left(A_{2} \underline{x}+\underline{f}_{n l}(\underline{x}, h(\underline{x}))\right)-\left(\lambda_{3} h(\underline{x})+g_{n l}(\underline{x}, h(\underline{x}))\right)
$$

with

$$
\frac{d h(\underline{x})}{d \underline{x}}=\left(\frac{\partial h}{\partial x_{1}}, \frac{\partial h}{\partial x_{2}}\right)
$$

Solutions of $h(\underline{x})$ can often be found by series expansion of $h$ in its variables $x_{1}$ and $x_{2}$, and since the constant and the linear part are already dealt with by the previous transformation one can start with quadratic terms, hence the ansatz

$$
h(\underline{x})=h_{20} x_{1}^{2}+h_{11} x_{1} x_{2}+h_{02} x_{2}^{2}+\mathcal{O}\left(\|\underline{x}\|^{3}\right)
$$

to be inserted into the equation $\mathcal{N}(h(\underline{x}))=0$.

No reference has been made yet to the scaling with the small parameter $\mu$. But by continuing to keep track of the scaling we would obtain the coefficients $h_{i j}$ as power series of $\mu$, hence

$$
h_{i j}=h_{0, i j}+\mu h_{1, i j}+\mu^{2} h_{2, i j}+\mathcal{O}\left(\mu^{3}\right)
$$

\subsection{Block structure transformation with real transformation matrix}

The analysis can be made easier by loosening the condition of exact diagonalizing of the Jacobian matrix to transformation into block structure, as e.g. excercised by $[3,4]$ in an SEIR epidemiological model for measles, going back to a first analysis in [5].

Observing the Jacobian matrix of the SIRUV around the endemic fixed point

$$
A=\left(\begin{array}{ccc}
-\frac{\beta}{M} \cdot V^{*}-\mu & 0 & -\frac{\beta}{M} \cdot S^{*} \\
\frac{\beta}{M} \cdot V^{*} & -(\gamma+\mu) & \frac{\beta}{M} \cdot S^{*} \\
0 & \frac{\vartheta}{N} \cdot\left(M-V^{*}\right) & -\frac{\vartheta}{N} I^{*}-\nu
\end{array}\right)
$$

gives for $\mu \rightarrow 0$ the matrix

$$
A_{0}=\left(\begin{array}{ccc}
0 & 0 & -\frac{\nu}{\vartheta} \gamma \frac{N}{M} \\
0 & -\gamma & \frac{\nu}{\vartheta} \gamma \frac{N}{M} \\
0 & \vartheta \frac{M}{N} & -\nu
\end{array}\right)
$$

by remembering that $I^{*}$ and $V^{*}$ are of order $\mu$ and $S^{*}=\frac{\gamma}{\frac{\vartheta}{\beta}} N+\mathcal{O}(\mu)$.

It will be useful later to dissect the matrix $A=A_{0}+A_{r}$ into the zero order part $A_{0}$ of the Jacobian matrix $A$ and the rest $A_{r}$ containing all higher orders of $\mu$ which can be easily done. 
The eigenvalues of the matrix $A_{0}$ are $\lambda_{1}=0, \lambda_{2}=0$ and $\lambda_{3}=-(\gamma+\nu)$, which corresponds to the zeroth order of the eigenvalues as we calculated in scaling before. Further, the eigenvectors of the lower right 2-dimensional square matrix are

$$
\underline{u}_{1}=\left(\begin{array}{c}
\frac{\nu}{\vartheta} \frac{N}{M} \\
1
\end{array}\right)
$$

and

$$
\underline{u}_{2}=\left(\begin{array}{c}
-\frac{\gamma}{\vartheta} \frac{N}{M} \\
1
\end{array}\right)
$$

Hence a real transformation matrix $\tilde{T}$ can be constructed via

$$
\tilde{T}=\left(\begin{array}{ccc}
1 & 0 & 0 \\
0 & \frac{\nu}{\vartheta} \frac{N}{M} & -\frac{\gamma}{\vartheta} \frac{N}{M} \\
0 & 1 & 1
\end{array}\right)=:\left(\begin{array}{ccc}
1 & 0 & 0 \\
0 & \varphi & \psi \\
0 & 1 & 1
\end{array}\right)
$$

which has as inverse

$$
\tilde{T}^{-1}=\frac{1}{\varphi-\psi}\left(\begin{array}{ccc}
\varphi-\psi & 0 & 0 \\
0 & 1 & -\psi \\
0 & -1 & \varphi
\end{array}\right)
$$

Then we have the transformation to block structure given by

$$
\begin{aligned}
\tilde{T}^{-1} A_{0} \tilde{T} & =\left(\begin{array}{ccc}
0 & -\frac{\nu}{\vartheta} \gamma \frac{N}{M} & -\frac{\nu}{\vartheta} \gamma \frac{N}{M} \\
0 & 0 & 0 \\
0 & 0 & -(\gamma+\nu)
\end{array}\right) \\
& =:\left(\begin{array}{ccc}
0 & \xi & \xi \\
0 & 0 & 0 \\
0 & 0 & -(\gamma+\nu)
\end{array}\right)=\left(\begin{array}{ccc}
A_{2} & \xi \\
0 & 0 & B
\end{array}\right)
\end{aligned}
$$

with

$$
\begin{aligned}
A_{2} & =\left(\begin{array}{ll}
0 & \xi \\
0 & 0
\end{array}\right) \\
B & =-(\gamma+\nu) \\
C & =\left(\begin{array}{l}
\xi \\
0
\end{array}\right)
\end{aligned}
$$

Now from

$$
\frac{d}{d t} \underline{z}=A \underline{z}+\underline{q}(\underline{z}, \mu)
$$

we have in zeroth order in $\mu$

$$
\frac{d}{d t} \underline{z}=A_{0} \underline{z}+\underline{q}(\underline{z}, \mu=0)
$$

and with $\underline{x}_{3}=\tilde{T}^{-1} \underline{z}$ we obtain the block structure

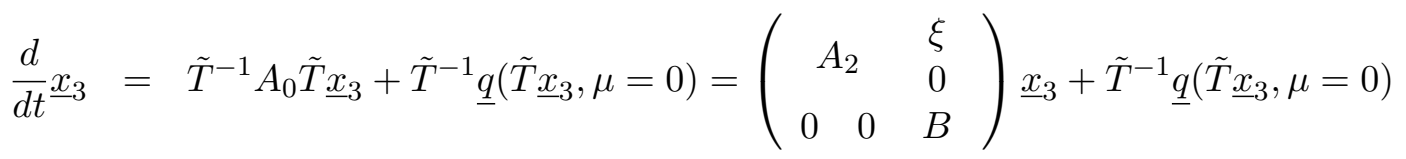


Hence in zeroth order in $\mu$ we have

$$
\begin{aligned}
\frac{d}{d t}\left(\begin{array}{l}
x_{1} \\
x_{2}
\end{array}\right) & =\left(\begin{array}{ll}
0 & \xi \\
0 & 0
\end{array}\right)\left(\begin{array}{l}
x_{1} \\
x_{2}
\end{array}\right)+\left(\begin{array}{l}
\xi \\
0
\end{array}\right) y+\underline{f}_{n l}(\underline{x}, y, \mu=0) \\
\frac{d}{d t} y & =-(\gamma+\nu) y+g_{n l}(\underline{x}, y, \mu=0)
\end{aligned}
$$

and with the blocks $A_{2}, B$ and $C$

$$
\begin{aligned}
& \frac{d}{d t} \underline{x}=A_{2} \underline{x}+C y+\underline{f}_{n l}(\underline{x}, y, \mu=0) \\
& \frac{d}{d t} y=B y+g_{n l}(\underline{x}, y, \mu=0)
\end{aligned}
$$

which has a structure for the center manifold analysis, see [9].

\subsection{Families of center manifolds}

Now for higher orders in $\mu$ we have to construct families of center manifolds [10] of the form

$$
\begin{aligned}
& \frac{d}{d t} \underline{x}=\underline{P}(\underline{x}, y, \mu) \\
& \frac{d}{d t} y=Q(\underline{x}, y, \mu)
\end{aligned}
$$

such that

$$
\begin{aligned}
& \underline{P}(\underline{x}, y, \mu=0)=A_{2} \underline{x}+C y+\underline{f}_{n l}(\underline{x}, y, \mu=0) \\
& Q(\underline{x}, y, \mu=0)=B y+g_{n l}(\underline{x}, y, \mu=0)
\end{aligned}
$$

We first have to calculate the explicit form of the general $\underline{P}$ and $Q$, e.g. from Eq. (22)

$$
\frac{d}{d t} \underline{z}=A \underline{z}+\underline{q}(\underline{z})=:\left(\begin{array}{c}
f_{1}(\underline{z}, \mu) \\
f_{2}(\underline{z}, \mu) \\
f_{3}(\underline{z}, \mu)
\end{array}\right)
$$

giving with $\underline{z}=\tilde{T} \underline{x}_{3}$ and $\underline{x}_{3}=\left(\begin{array}{c}\underline{x} \\ y\end{array}\right)=\left(\begin{array}{c}x_{1} \\ x_{2} \\ y\end{array}\right)$ the expressions for $\underline{P}$ and $Q$

$$
\frac{d}{d t} \underline{x}_{3}=\tilde{T}^{-1}\left(\begin{array}{c}
f_{1}\left(\tilde{T} \underline{x}_{3}, \mu\right) \\
f_{2}\left(\tilde{T} \underline{x}_{3}, \mu\right) \\
f_{3}\left(\tilde{T} \underline{x}_{3}, \mu\right)
\end{array}\right)=\left(\begin{array}{c}
\underline{P}(\underline{x}, y, \mu) \\
Q(\underline{x}, y, \mu)
\end{array}\right)=\frac{d}{d t}\left(\begin{array}{c}
\underline{x} \\
y
\end{array}\right)
$$


This gives us the families of center manifolds with parameter $\mu$

$$
y=w(\underline{x}, \mu)
$$

to be determined from

$$
\frac{d w(\underline{x}, \mu)}{d \underline{x}} \cdot \underline{P}(\underline{x}, w(\underline{x}, \mu), \mu)-Q(\underline{x}, w(\underline{x}, \mu), \mu)=0
$$

via series expansion

$$
w(\underline{x}, \mu)=w_{0}(\underline{x})+\mu \cdot w_{1}(\underline{x})+\mu^{2} \cdot w_{2}(\underline{x})+\mathcal{O}\left(\mu^{3}\right)
$$

Especially we have for the center manifold in zeroth order $y=h(\underline{x})$ from the equation system, Eq. (45),

$$
w(\underline{x}, \mu=0)=h(\underline{x})
$$

see $[10]$.

\subsection{Results for the SIRUV model}

For the SIRUV model we have explicitly for

$$
\begin{aligned}
\frac{d}{d t} \underline{z} & =A \underline{z}+\underline{q}(\underline{z}) \\
& =\left(A_{0}+A_{r}\right) \underline{z}+\left(\begin{array}{c}
-\frac{\beta}{M} z_{1} z_{3} \\
\frac{\beta}{M} z_{1} z_{3} \\
-\frac{\vartheta}{N} z_{3} z_{2}
\end{array}\right)
\end{aligned}
$$

in which the Jacobian matrix $A$ is decomposed into its zeroth order component $A_{0}$ and the remaining $\mu$-dependent rest $A_{r}=A_{r}(\mu)$. Also it should be noted that the non-linear part $\underline{q}(\underline{z})$ is not dependent on $\mu$. Explicitly, we have for $A=A_{0}+A_{r}$ the result

$$
A=\left(\begin{array}{ccc}
0 & 0 & -\frac{\nu}{\vartheta} \gamma \frac{N}{M} \\
0 & -\gamma & \frac{\nu}{\vartheta} \gamma \frac{N}{M} \\
0 & \vartheta \frac{M}{N} & -\nu
\end{array}\right)+\left(\begin{array}{ccc}
-\frac{\beta}{M} \cdot V^{*}-\mu & 0 & -\mu\left(\frac{\nu}{\vartheta}+\frac{\beta}{\beta+\mu}\right) \frac{N}{M} \\
\frac{\beta}{M} \cdot V^{*} & -\mu & \mu\left(\frac{\nu}{\vartheta}+\frac{\beta}{\beta+\mu}\right) \frac{N}{M} \\
0 & -\frac{\vartheta}{N} \cdot V^{*} & -\frac{\vartheta}{N} I^{*}
\end{array}\right)
$$

With the transformation matrix $\tilde{T}$ we then obtain

$$
\frac{d}{d t}\left(\begin{array}{c}
x_{1} \\
x_{2} \\
y
\end{array}\right)=\frac{d}{d t} \underline{x}_{3}=\left(\tilde{T}^{-1} A_{0} \tilde{T}+\tilde{T}^{-1} A_{r} \tilde{T}\right) \underline{x}_{3}+\tilde{T}^{-1} \underline{q}\left(\tilde{T} \underline{x}_{3}\right)=\left(\begin{array}{c}
P_{1} \\
P_{2} \\
Q
\end{array}\right)
$$

with the zeroth order terms

$$
\tilde{T}^{-1} A_{0} \tilde{T} \underline{x}_{3}=\left(\begin{array}{ccc}
0 & \xi & \xi \\
0 & 0 & 0 \\
0 & 0 & -(\gamma+\nu)
\end{array}\right) \cdot \underline{x}_{3}=\left(\begin{array}{c}
\xi\left(x_{2}+y\right) \\
0 \\
-(\gamma+\nu) y
\end{array}\right)
$$


and

$$
\tilde{T}^{-1} \underline{q}\left(\tilde{T} \underline{x}_{3}\right)=\tilde{T}^{-1}\left(\begin{array}{c}
-\frac{\beta}{M} x_{1} \\
\frac{\beta}{M} x_{1} \\
-\frac{\vartheta}{N}\left(\varphi x_{2}+\psi y\right)
\end{array}\right) \cdot\left(x_{2}+y\right)=\left(\begin{array}{c}
\tilde{f}_{1, n l} \\
\tilde{f}_{2, n l} \\
\tilde{g}_{n l}
\end{array}\right) \cdot\left(x_{2}+y\right)
$$

and the linear term of order $\mu$

$$
\begin{aligned}
\tilde{T}^{-1} A_{r} \tilde{T} \underline{x}_{3}= & \left(\begin{array}{c}
\left(-\frac{\beta}{M} \cdot V^{*}-\mu\right) x_{1}-\mu\left(\frac{\nu}{\vartheta}+\frac{\beta}{\beta+\mu}\right) \frac{N}{M}\left(x_{2}+y\right) \\
\frac{1}{\varphi-\psi}\left[\frac{\beta}{M} \cdot V^{*} x_{1}+\left(-\mu+\psi \frac{\vartheta}{N} \cdot V^{*}\right)\left(\varphi x_{2}+\psi y\right)\right. \\
\left.+\left(\mu\left(\frac{\nu}{\vartheta}+\frac{\beta}{\beta+\mu}\right) \frac{N}{M}+\psi \frac{\vartheta}{N} \cdot I^{*}\right)\left(x_{2}+y\right)\right] \\
\frac{1}{\varphi-\psi}\left[-\frac{\beta}{M} \cdot V^{*} x_{1}+\left(\mu-\varphi \frac{\vartheta}{N} \cdot V^{*}\right)\left(\varphi x_{2}+\psi y\right)\right. \\
\left.+\left(-\mu\left(\frac{\nu}{\vartheta}+\frac{\beta}{\beta+\mu}\right) \frac{N}{M}-\varphi \frac{\vartheta}{N} \cdot I^{*}\right)\left(x_{2}+y\right)\right]
\end{array}\right) \\
= & \mu\left(\begin{array}{c}
r_{11} x_{1}+r_{12} x_{2}+r_{13} y \\
r_{21} x_{1}+r_{22} x_{2}+r_{23} y \\
r_{31} x_{1}+r_{32} x_{2}+r_{33} y
\end{array}\right)+\mathcal{O}\left(\mu^{2}\right)=: \mu \mathcal{R} \underline{x}_{3}+\mathcal{O}\left(\mu^{2}\right)
\end{aligned}
$$

\subsubsection{Results for the SIRUV model in lowest order $\mathcal{O}\left(\mu^{0}\right)$}

We have in zeroth order in $\mu$

$$
\frac{d}{d t}\left(\begin{array}{c}
x_{1} \\
x_{2} \\
y
\end{array}\right)=\left(\begin{array}{c}
\xi\left(x_{2}+y\right) \\
0 \\
-(\gamma+\nu) y
\end{array}\right)+\tilde{T}^{-1}\left(\begin{array}{c}
-\frac{\beta}{M} x_{1} \\
\frac{\beta}{M} x_{1} \\
-\frac{\vartheta}{N}\left(\varphi x_{2}+\psi y\right)
\end{array}\right) \cdot\left(x_{2}+y\right)
$$

hence for $y=h\left(x_{1}, x_{2}\right)$ we have the equation

$$
\left(\frac{\partial h}{\partial x_{1}}, \frac{\partial h}{\partial x_{2}}\right)\left[\xi\left(\begin{array}{l}
1 \\
0
\end{array}\right)+\underline{\tilde{f}}(\underline{x}, h)\right]\left(x_{2}+h\right)+\left[(\gamma+\nu) h-\tilde{g}(\underline{x}, h)\left(x_{2}+h\right)\right]=0
$$

and with the ansatz in the leading quadratic terms

$$
h\left(x_{1}, x_{2}\right)=h_{20} x_{1}^{2}+h_{11} x_{1} x_{2}+h_{02} x_{2}^{2}+\mathcal{O}\left(\|\underline{x}\|^{3}\right)
$$

and after inserting and comparing the terms in $x_{1}^{2}, x_{1} x_{2}$ and $x_{2}^{2}$ we obtain

$$
h\left(x_{1}, x_{2}\right)=h_{11} \cdot x_{1} x_{2}+h_{02} \cdot x_{2}^{2}+\mathcal{O}\left(\|\underline{x}\|^{3}\right)
$$

with $h_{20}=0, h_{02}=-\left(\frac{\nu}{\gamma+\nu}\right)^{2} \frac{1}{M}-\frac{\beta \gamma \nu}{(\gamma+\nu)^{3}} \frac{1}{M}$ and $h_{11}=-\frac{\vartheta \beta}{(\gamma+\nu)^{2}} \frac{1}{N}$.

\subsubsection{Results for the SIRUV model in next to leading order $\mathcal{O}(\mu)$}

In higher order in $\mu$ we have

$$
\frac{d}{d t}\left(\begin{array}{c}
x_{1} \\
x_{2} \\
y
\end{array}\right)=\left(\begin{array}{c}
\xi\left(x_{2}+y\right) \\
0 \\
-(\gamma+\nu) y
\end{array}\right)+\tilde{T}^{-1}\left(\begin{array}{c}
-\frac{\beta}{M} x_{1} \\
\frac{\beta}{M} x_{1} \\
-\frac{\vartheta}{N}\left(\varphi x_{2}+\psi y\right)
\end{array}\right) \cdot\left(x_{2}+y\right)+\mu \mathcal{R} \underline{x}_{3}+\mathcal{O}\left(\mu^{2}\right)=\left(\begin{array}{c}
P_{1} \\
P_{2} \\
Q
\end{array}\right)
$$


and hence for the family of center manifolds $y=w(\underline{x}, \mu)$ we have

$$
\frac{d w(\underline{x}, \mu)}{d \underline{x}} \underline{P}-Q=0
$$

Further for $w$ we have the ansatz

$$
\begin{aligned}
y & =w(\underline{x}, \mu)=w_{0}+\mu w_{1}+\mathcal{O}\left(\mu^{2}\right) \\
& =\left(h_{02} x_{2}^{2}+h_{11} x_{1} x_{2}+\mathcal{O}\left(\|\underline{x}\|^{3}\right)\right)+\mu w_{1}(\underline{x})+\mathcal{O}\left(\mu^{2}\right)
\end{aligned}
$$

with $w_{0}(\underline{x})=h(\underline{x})$, and $h$ as calculated before in zeroth order.

Now in approximation up to first order in $\mu$ with

$$
\begin{aligned}
w(\underline{x}, \mu) & =w_{0}+\mu w_{1}=h(\underline{x})+\mu w_{1}(\underline{x}) \\
\underline{P} & =\underline{P}_{0}+\mu \underline{P}_{1} \\
Q & =Q_{0}+\mu Q_{1}
\end{aligned}
$$

we have

$$
\frac{d\left(w_{0}+\mu w_{1}\right)}{d \underline{x}}\left(\underline{P}_{0}+\mu \underline{P}_{1}\right)-\left(Q_{0}+\mu Q_{1}\right)=0
$$

or

$$
\underbrace{\left(\frac{d h}{d \underline{x}} \underline{P}_{0}-Q_{0}\right)}_{=: D}+\mu \frac{d h}{d \underline{x}} \underline{P}_{1}+\mu \frac{d w_{1}}{d \underline{x}} \underline{P}_{0}-\mu Q_{1}=0
$$

where we already know from the zeroth order calculations above that the leading term is $\left(\frac{d h}{d \underline{x}} \underline{P}_{0}(h)-Q_{0}(h)\right)=0$ and that

$$
\frac{d h(\underline{x})}{d \underline{x}}=\left(\frac{\partial h}{\partial x_{1}}, \frac{\partial h}{\partial x_{2}}\right)=\left(h_{11} x_{2}, 2 h_{02} x_{2}+h_{11} x_{1}\right)+\mathcal{O}\left(\|\underline{x}\|^{3}\right)
$$

and finally

$$
\underline{P}_{0}=\left(\xi\left(\begin{array}{l}
1 \\
0
\end{array}\right)+\underline{\tilde{f}}(\underline{x}, h)\right)\left(x_{2}+h_{02} x_{2}^{2}+h_{11} x_{1} x_{2}\right)+\mathcal{O}\left(\|\underline{x}\|^{3}\right)
$$

Further we have with $A=A_{0}+A_{r}=A_{0}+\mu A_{1}+\mu^{2} A_{2}+\mathcal{O}\left(\mu^{3}\right)$

$$
\begin{aligned}
& \left(\begin{array}{c}
\underline{P}_{1} \\
Q_{1}
\end{array}\right)=\tilde{T}^{-1} A_{1} \tilde{T} \underline{x}_{3}=\left(\begin{array}{c}
P_{1,1} \\
P_{2,1} \\
Q_{1}
\end{array}\right) \\
& =\tilde{T}^{-1}\left(\begin{array}{ccc}
-\frac{\frac{\vartheta}{\nu} \beta}{\gamma} & 0 & -\left(\frac{\nu}{\vartheta}+1-\frac{\gamma}{\frac{\vartheta}{\nu} \beta}\right) \frac{M}{N} \\
\frac{\vartheta}{\nu} \beta & -1 & \left(\frac{\nu}{\vartheta}+1-\frac{\gamma}{\frac{\vartheta}{\nu} \beta}\right) \frac{M}{N} \\
0 & -\frac{\vartheta^{2}}{\nu \gamma}\left(1-\frac{\gamma}{\frac{\vartheta}{\nu} \beta}\right) \frac{M}{N} & -\frac{\vartheta}{\gamma}\left(1-\frac{\gamma}{\frac{\vartheta}{\nu} \beta}\right)
\end{array}\right) \tilde{T} \underline{x}_{3} \\
& =: \quad \tilde{T}^{-1}\left(\begin{array}{ccc}
-\alpha_{11} & 0 & -\alpha_{23} \\
\alpha_{11}-1 & -1 & \alpha_{23} \\
0 & -\alpha_{32} & -\alpha_{33}
\end{array}\right) \tilde{T} \underline{x}_{3}=\mathcal{R} \underline{x}_{3}
\end{aligned}
$$


With this we have fully determined the terms in $\mathcal{O}(\mu)$ in

$$
\frac{d h}{d \underline{x}} \underline{P}_{1}+\frac{d w_{1}}{d \underline{x}} \underline{P}_{0}-Q_{1}+D=0
$$

or in full as determining equation for $w_{1}(\underline{x})$ from

$$
\frac{d h}{d \underline{x}} \underline{P}_{1}\left(\underline{x}, h(\underline{x})+\mu w_{1}(\underline{x})\right)+\frac{d w_{1}}{d \underline{x}} \underline{P}_{0}\left(\underline{x}, h(\underline{x})+\mu w_{1}(\underline{x})\right)-Q_{1}\left(\underline{x}, h(\underline{x})+\mu w_{1}(\underline{x})\right)+D=0
$$

we simply have

$$
\frac{d h}{d \underline{x}} \underline{P}_{1}(\underline{x}, h(\underline{x}))+\frac{d w_{1}}{d \underline{x}} \underline{P}_{0}(\underline{x}, h(\underline{x}))-Q_{1}(\underline{x}, h(\underline{x}))+D=0
$$

as next to leading order, since from Eq. (64) we see that Eq. (69) is already in first order in $\mu$.

\subsubsection{Backtransformation to original coordinates and parameters in leading order}

We have the center manifold in zeroth order as $y=h(\underline{x})=h_{02} x_{2}^{2}+h_{11} x_{1} x_{2}$. Closer to the original coordinates $z_{3}=V(t)-V^{*}$ we have with the transformation $\underline{z}=\tilde{T} \underline{x}_{3}=\left(x_{1}, \phi x_{2}+\psi y, x_{2}+y\right)^{t r}$, hence $z_{3}=x_{2}+y=x_{2}+h_{02} x_{2}^{2}+$ $h_{11} x_{1} x_{2}=z_{3}\left(x_{1}, x_{2}\right)$, hence $z_{3}=V(t)-V^{*}$ as a function of $x_{2}$. And from $\underline{x}_{3}=$ $\tilde{T}^{-1} \underline{z}=\left(z_{1}, \frac{1}{\varphi-\psi}\left(z_{2}-\psi z_{3}\right), \frac{1}{\varphi-\psi}\left(-z_{2}+\varphi z_{3}\right)\right)^{t r}$, hence $x_{2}=\frac{1}{\varphi-\psi}\left(z_{2}-\psi z_{3}\right)$ we can insert to obtain $z_{3}\left(z_{2}\right)$, hence $z_{3}=V(t)-V^{*}$ as a function of $z_{2}=I(t)-I^{*}$. Again cutting short, in lowest order we have $z_{3}=x_{2}=\frac{1}{\varphi-\psi}\left(z_{2}-\psi z_{3}\right)$ and hence

$$
z_{3}=\frac{1}{\varphi} z_{2}=\frac{\vartheta}{\nu} \frac{M}{N} z_{2}
$$

which gives

$$
V(t)=\frac{\vartheta}{\nu} \frac{M}{N} I(t)
$$

as final result. Eq. (72) hence gives in lowest order in $\mu$ the result $V(I)$ proportional to $I$, which results in effective SIR-type models for the human infecteds only.

For the SISUV model in contrast, see Appendix B, we obtain with Eq. (147) in lowest order, now of $\varepsilon$, the Holling type II form for $V(I)$, hence

$$
V(t)=\frac{\frac{\vartheta}{\nu} I(t)}{N+\frac{\vartheta}{\nu} I(t)} \cdot M
$$

since in the SISUV model $I^{*}$ and hence for long times $I(t)$ is of order one and not of order $\varepsilon$. The simplification

$$
V(t)=\frac{\frac{\vartheta}{\nu} I(t)}{N+\frac{\vartheta}{\nu} I(t)} \cdot M \rightarrow \frac{\vartheta}{\nu} \frac{M}{N} I(t)
$$

for biologically small parameter $\mu \rightarrow 0$ holds for the SIRUV model, if obtained by any method (e.g. as quasi-steady state assumption QSSA, see [2]), but not for the SISUV model. The present center manifold analysis of the SIRUV model gives in lowest order directly the simple linear relation. 


\section{Acknowledgments}

M. A. has received funding from the European Unions Horizon 2020 research and innovation programme under the Marie Skłodowska-Curie grant agreement No 792494. This research is also supported by the Basque Government through BERC 2018-2021 program and by Spanish Ministry of Sciences, Innovation and Universities: BCAM Severo Ochoa accreditation SEV-2017-0718. N.St. thanks the Mathematics Department of Trento University for the opportunity and support as guest lecturer in biomathematics, during which part of the present work has been conceived and started to be developed.

\section{References}

[1] Rocha, F., Aguiar, M., Souza, M., \& Stollenwerk, N. (2013) Time-scale separation and center manifold analysis describing vector-borne disease dynamics, Int. Journal. Computer Math. 90, 2105-2125.

[2] Rashkov, P., Venturino, E., Aguiar, M., Stollenwerk, N., \& Kooi, B. (2019) On the role of vector modelling in a minimalistic epidemiological model, Mathematical Biosciences and Engineering 16, 4314-4338.

[3] Forgoston, E., Billings, L., \& Schwartz, I.B. (2019) Model Reduction in Stochastic Environments, pp. 37-62, in Wei, W., Xiaopeng, C., \& Yan, L. (eds.) Stochastic PDEs and Modelling of Multiscale Complex Systems, World Scientific, Singapore, (arXiv:1711.07842v1, 20 Nov. 2017).

[4] Forgoston, E., Billings, L., \& Schwartz, I.B. (2009) Accurate noise projection for reduced stochastic epidemic models, Chaos 19, 043110.

[5] Schwartz, I.B., \& Smith, H. (1983), Infinite subharmonic bifurcations in an SEIR epidemic model, J. Math. Biol. 18, 233-253.

[6] Benoît, E., Brons, M., Desroches, M., \& Krupa, M. (2015) Extending the zero-derivative principle for slowfast dynamical systems, J. Zeitschrift fuer Angewandte Mathematik und Physik, 66, 2255-2270.

[7] Kaper, H.G., \& Kaper, T.J. (2002) Asymptotic analysis of two reduction methods for systems of chemical reactions. Physica D: Nonlinear Phenomena, 165 66-93.

[8] Farrés, A., \& Jorba, A. (2010) On the higher order approximation of the center manifold for ODEs, Discrete and Continuous Dynamical Systems, Series B 14, 977-1000.

[9] Schecter, S. (2002) Traveling-wave solutions of convection-diffusion systems by center manifold reduction, Nonlinear Analysis 49, 35-59.

[10] Kuznetsov, Y.A. (2010) Elements of applied bifurcation theory, SpringerVerlag, New York. 
[11] Rocha, F., Mateus, L., Skwara, U., Aguiar, M., \& Stollenwerk, N. (2016) Understanding dengue fever dynamics: a study of seasonality in vector borne disease models, International Journal of Computer Mathematics, 93, $1405-1422$.

[12] Jardón-Kojakhmetov, H., Kühn, Ch., Pugliese, A. \& Sensi, M. (2019) A geometric analysis of SIR, SIRS and SIRWS epidemiological models, arXiv:2002.00354v1, 4 Feb. 2020. 


\section{A Eigenvalues of the Jacobian matrix around the endemic fixed point via Cardano's method and its scaling}

\section{A.1 Cardano's method for finding the zeros of 3rd order poly- nomials}

For any polynomial

$$
0=x^{3}+a_{2} x^{2}+a_{1} x+a_{0}
$$

we can solve the equation by first reducing it via a coordinate transformation

$$
z:=x+\frac{1}{3} a_{2}
$$

to the form

$$
0=z^{3}+p z+q
$$

with

and

$$
p=a_{1}-\frac{1}{3} a_{2}^{2}
$$

$$
q=\frac{2}{27} a_{2}^{3}-\frac{1}{3} a_{1} a_{2}+a_{0}
$$

and further express $z$ as sum of two variables $u$ and $v$, hence

$$
z=: u+v
$$

which gives the 3rd order polynomial in the form

$$
0=\left(u^{3}+v^{3}+q\right)+(3 u v+p)(u+v)
$$

From this we get via setting the second bracket expression to zero the variable $v$ as

$$
v=-\frac{p}{3 u}
$$

and setting th first bracket expression to zero we obtain for the variable $u^{3}$ a solvable quadratic equation

$$
0=\left(u^{3}\right)^{2}+q u^{3}-\left(\frac{p}{3}\right)^{3}
$$

Hence we have

$$
u^{3}=-\frac{q}{2}+\sqrt{\frac{1}{4} q^{2}+\frac{1}{27} p^{3}}=: k
$$

and $s:=\frac{1}{4} q^{2}+\frac{1}{27} p^{3}$.

Then the cubic Eq. (84) has three solutions with a phase factor $\tilde{\psi}=e^{i \cdot \frac{2}{3} \pi}$, namely

$$
u_{1}=k^{\frac{1}{3}} \quad, \quad u_{2}=\tilde{\psi} \cdot u_{1} \quad, \quad u_{3}=\tilde{\psi}^{2} \cdot u_{1}
$$

and their respective solutions $v_{j}=-\left(p / 3 u_{j}\right)$, hence $x_{j}=u_{j}+v_{j}-a_{2} / 3$ for $j=1,2,3$. We have as powers of the phase factor $\tilde{\psi}=e^{i \cdot \frac{2}{3} \pi}=-\frac{1}{2}+\frac{1}{2} \sqrt{3} i$ and $\tilde{\psi}^{2}=e^{i \cdot \frac{4}{3} \pi}=-\frac{1}{2}-\frac{1}{2} \sqrt{3} i$, and of course $\tilde{\psi}^{3}=e^{i \cdot \frac{6}{3} \pi}=e^{i \cdot 2 \pi}=1$. 


\section{A.2 Application of Cardano's method to the characteristic poly- nomial of the SIRUV Jacobian matrix}

Implementing Cardano's method for the parameters given in the SIRUV model and its Jacobian matrix we find

$$
\begin{aligned}
a_{2} & =88.7359 \\
a_{1} & =6.2950 \\
a_{0} & =87.8320 \\
p & =-2618.3905 \\
q & =51658.0299 \\
s & =2263806.0978 \quad>0 \\
\sqrt{s} & =1504.5950 \\
k & =-24324.419973 \quad \text { hence a real number } \\
u:=k^{1 / 3} & =-28.9744 \quad \text { hence a real number } \\
v:=-(p / 3 u) & =-30.1231 \quad \text { hence a real number } \\
x:=u+v-a_{2} / 3 & =-88.67601 \quad=c \text { hence a real number } \\
x_{r}:=-(u / 2)-(v / 2)-a_{2} / 3 & =-0.029909 \quad=a \\
x_{i}:=\sqrt{3}(u / 2)-\sqrt{3}(v / 2)-a_{2} / 3 & =0.994780 \quad=b
\end{aligned}
$$

and hence gives the numerical values of the eigenvalues as follows

$$
\lambda_{1 / 2}=a \pm i \cdot b=x_{r} \pm i \cdot x_{i}=-0.029909 \pm i \cdot 0.994780
$$

and

$$
\lambda_{3}=c=x=-88.67601
$$

in good numerical agreement with [1], where the SRV submodel was used, and not as here the SIV submodel.

Now that we have the analytic form of the eigenvalues implicitly given via Cardano's method, we can investigate the scaling of all relevant quantities with the small parameter $\mu$. This will be done in the next section.

\section{A.3 Scaling with small parameter $\mu$}

First we observe numerically the scaling of all relevant quantities with the parameter $\mu$ by decreasing $\mu$ by a factor of 10 and comparing with the previous 
results from the original value of $\mu$, hence

$$
\begin{aligned}
a_{2} & =88.6522=\mathcal{O}(1) \\
a_{1} & =0.6297=\mathcal{O}(\mu) \\
a_{0} & =8.7840=\mathcal{O}(\mu) \\
p & =-2619.1057=\mathcal{O}(1) \\
q & =51600.2234=\mathcal{O}(1) \\
s & =226579.5178=\mathcal{O}(\mu) \\
\sqrt{s} & =476.0037=\mathcal{O}(\sqrt{\mu}) \\
k & =-25324.1080=\mathcal{O}(1) \\
u:=k^{1 / 3} & =-29.365995=\mathcal{O}(1) \\
v:=-(p / 3 u) & =-29.729462=\mathcal{O}(1) \\
x:=u+v-a_{2} / 3 & =-88.646179=c \quad=\mathcal{O}(1) \\
x_{r}:=-(u / 2)-(v / 2)-a_{2} / 3 & =-0.002993 \quad=a \quad=\mathcal{O}(\mu) \\
x_{i}:=\sqrt{3}(u / 2)-\sqrt{3}(v / 2)-a_{2} / 3 & =0.314772 \quad=b \quad=\mathcal{O}(\sqrt{\mu})
\end{aligned}
$$

with many parameters of the Cardano method like $p, q, u$ and $v$ of order $\mathcal{O}(1)$, while the results $a=-(u / 2)-(v / 2)-a_{2} / 3=\mathcal{O}(\mu)$ and $b=\sqrt{3}(u / 2)-$ $\sqrt{3}(v / 2)-a_{2} / 3=\mathcal{O}(\sqrt{\mu})$ are of higher orders in $\mu$.

Hence any scaling analysis has to take at least orders of $\mu$ into account, since order-one parameters in subtractions cancel the lowest order, and leave higher orders in $\mu$ only.

\section{A.4 Analytical results of the scaling analysis with small param- eter $\mu$}

\section{A.4.1 Scaling of endemic stationary state}

For the stationary state we have the following scaling up to first order in $\mu$

$$
\frac{S^{*}}{N}=\frac{\nu \gamma}{\vartheta \beta}+\frac{\nu+\vartheta-\nu \frac{\gamma}{\beta}}{\vartheta \beta} \mu+\mathcal{O}\left(\mu^{2}\right)=\mathcal{O}(1)
$$

and

$$
\frac{I^{*}}{N}=\frac{\mu}{\gamma}\left(1-\frac{\gamma}{\frac{\vartheta}{\nu} \beta}\right)+\mathcal{O}\left(\mu^{2}\right) \quad=\mathcal{O}(\mu)
$$

and

$$
\frac{V^{*}}{M}=\frac{\vartheta}{\nu} \frac{I^{*}}{N}+\mathcal{O}\left(I^{* 2}\right) \quad=\mathcal{O}(\mu)
$$




\section{A.4.2 Scaling of characteristic polynomial coefficients}

The coefficients of the characteristic polynomial $0=\lambda^{3}+a_{2} \lambda^{2}+a_{1} \lambda+a_{0}$ are in scaling up to first order in $\mu$ given by

$$
\begin{aligned}
a_{2} & =-\left(a_{11}+a_{22}+a_{33}\right) \\
& =\gamma+\nu+\frac{\vartheta}{N} I^{*}+\frac{\beta}{M} \cdot V^{*}+2 \mu \\
& =(\gamma+\nu)+\left(1+\frac{\vartheta \beta}{\nu \gamma}+\frac{\vartheta}{\gamma}-\frac{\nu}{\beta}\right) \mu+\mathcal{O}\left(\mu^{2}\right) \\
a_{1} & =a_{11} a_{22}+a_{33}\left(a_{11}+a_{22}\right)-a_{23} a_{32} \\
& =(\gamma+\mu)\left(\frac{\beta}{M} \cdot V^{*}+\mu\right)+\left(\nu+\frac{\vartheta}{N} I^{*}\right)\left(\gamma+\frac{\beta}{M} \cdot V^{*}+2 \mu\right)-\vartheta \beta \frac{S^{*}}{N}\left(1-\frac{V^{*}}{M}\right) \\
& =\left((\gamma+\nu) \cdot \frac{\vartheta \beta}{\nu \gamma}+\vartheta-\nu \frac{\gamma}{\beta}\right) \mu+\mathcal{O}\left(\mu^{2}\right) \\
a_{0} & =-a_{11} a_{22} a_{33}-\mu a_{23} a_{32} \\
& =(\gamma+\mu)\left(\nu+\frac{\vartheta}{N} I^{*}\right)\left(\frac{\beta}{M} \cdot V^{*}+\mu\right) \\
& =(\vartheta \beta-\nu \gamma) \mu+\mathcal{O}\left(\mu^{2}\right)
\end{aligned}
$$

and are numerically tested.

\section{A.4.3 Scaling of eigenvalues}

For the scaling of the eigenvalues we need to take quantities like $c_{\mu}:=a_{2}+c$ into account, where $a_{2}=\mathcal{O}(1)$ and $c=\mathcal{O}(1)$ but remembering that $a_{2}$ is positive and $c$ is negative we obtain $c_{\mu}=\mathcal{O}(\mu)$. And for this we have to determine the order of $\lambda_{3}=c$ from the whole Cardano procedure.

Once $c$ is known, we can perform a polynomial divison of the characteristic polynomial $0=\lambda^{3}+a_{2} \lambda^{2}+a_{1} \lambda+a_{0}$ obtaining

$$
0=(\lambda-c) \cdot\left(\lambda^{2}+\left(a_{2}+c\right) \lambda+c\left(a_{2}+c\right)+a_{1}\right)
$$

such that we then easily obtain the higher order quantities of the complex conjugated eigenvalues $\lambda_{1 / 2}=a \pm i \cdot b$ as

$$
\lambda_{1 / 2}=-\frac{1}{2}\left(a_{2}+c\right) \pm \sqrt{\frac{1}{4}\left(a_{2}+c\right)^{2}-\left(c\left(a_{2}+c\right)+a_{1}\right)}=a \pm i \cdot b
$$

With the numerical values of $a_{2}=88.735880$ and from Cardano's method $c=x=-88.676061$ we obtain numerically $c_{\mu}=a_{2}+c=0.059819$, hence we have via the polynomial division

$$
a=-\frac{1}{2} c_{\mu}=-0.029909
$$

in complete agreement with the result from Cardano's method. Further we can approximate $b$ to order $\mathcal{O}\left(\mu^{1 / 2}\right)$ as

$$
b=\sqrt{-a_{2} c_{\mu}+a_{1}}+\mathcal{O}(\mu)=\mathcal{O}\left(\mu^{1 / 2}\right)=0.993430+\mathcal{O}(\mu)
$$


also in good agreement with the result from Cardano's method of $b=x_{i}=$ 0.994780 . And scaling can be checked again by decreasing $\mu$ by a factor of 100 , hence $\mathcal{O}\left(\mu^{1 / 2}\right)$ gives a factor of 10 , with the result of $b=x_{i}=0.099546$ and its approximation $b=\sqrt{-a_{2} c_{\mu}+a_{1}}=0.099544$.

The result of the scaling analysis for $c_{\mu}$ through out Cardano's method is

$$
c_{\mu}:=a_{2}+c=-\frac{a_{0}}{a_{2}^{2}}+\frac{a_{1}}{a_{2}}+\mathcal{O}\left(\mu^{3 / 2}\right)=\mathcal{O}\left(\mu^{1}\right)
$$

or with $-\frac{a_{0}}{a_{2}^{2}}+\frac{a_{1}}{a_{2}}=\left(a_{2} \cdot a_{1}-a_{0}\right) / a_{2}^{2}$ we have

$$
c_{\mu}=\frac{1}{(\gamma+\nu)^{2}}\left((\gamma+\nu) \cdot \tilde{a}_{1}-\tilde{a}_{0}\right)+\mathcal{O}\left(\mu^{3 / 2}\right)
$$

with $a_{1}=\tilde{a}_{1}+\mathcal{O}\left(\mu^{2}\right)$ and $a_{0}=\tilde{a}_{0}+\mathcal{O}\left(\mu^{2}\right)$, hence

$$
\tilde{a}_{1}=\left((\gamma+\nu) \cdot \frac{\vartheta \beta}{\nu \gamma}+\vartheta-\nu \frac{\gamma}{\beta}\right) \mu
$$

and

$$
\tilde{a}_{0}=(\vartheta \beta-\nu \gamma) \mu .
$$

Or completely in terms of the original model parameters we have the final result of

$$
c_{\mu}=\left(\frac{\vartheta \beta}{\nu \gamma}+\frac{\vartheta-\nu \frac{\gamma}{\beta}}{\gamma+\nu}-\frac{\vartheta \beta-\nu \gamma}{(\gamma+\nu)^{2}}\right) \cdot \mu+\mathcal{O}\left(\mu^{3 / 2}\right)
$$

which gives the result of $c_{\mu}=0.05981865+\mathcal{O}\left(\mu^{3 / 2}\right)$ as compared to the exact result $c_{\mu}=a_{2}+c=0.05986159$.

Further we have the original 3rd eigenvalue given as

$$
\lambda_{3}=c=-a_{2}+c_{\mu}=-(\gamma+\nu)-\left(1+\frac{\vartheta}{\gamma}-\frac{\nu}{\beta}-\frac{\vartheta-\nu \frac{\gamma}{\beta}}{\gamma+\nu}+\frac{\vartheta \beta-\nu \gamma}{(\gamma+\nu)^{2}}\right) \cdot \mu+\mathcal{O}\left(\mu^{3 / 2}\right)
$$

and the other two parameters of the first two eigenvalues $\lambda_{1 / 2}=a \pm i b$ are

$$
a=-\frac{1}{2} c_{\mu}+\mathcal{O}\left(\mu^{3 / 2}\right)
$$

trivially and further

$$
b=\sqrt{-a_{2} \cdot c_{\mu}-a_{1}}+\mathcal{O}\left(\mu^{3 / 2}\right)=\sqrt{\frac{\vartheta \beta-\nu \gamma}{\gamma+\nu}} \cdot \sqrt{\mu}+\mathcal{O}\left(\mu^{3 / 2}\right)
$$

and numerically $b=0.995465+\mathcal{O}\left(\mu^{3 / 2}\right)$ as compared to the exact result from Cardano's method of $b=0.994780$. And as check of the scaling when decreasing $\mu$ again by a factor of 100 , we obtain as approximation $b=0.09954648+\mathcal{O}\left(\mu^{3 / 2}\right)$ as compared to the exact $b=0.09954580$. 


\section{B Center manifold analysis for the SISUV model with scaling analysis}

The SISUV model is given by

$$
\begin{aligned}
\frac{d}{d t} S & =\alpha I-\frac{\beta}{M} S V \\
\frac{d}{d t} I & =\frac{\beta}{M} S V-\alpha I \\
\frac{d}{d t} U & =\psi-\nu U-\frac{\vartheta}{N} U I \\
\frac{d}{d t} V & =\frac{\vartheta}{N} U I-\nu V
\end{aligned}
$$

with all parameters as also used in the SIRUV model, but $\alpha=\frac{1}{10 y}$ and $\beta=2 \cdot \alpha$, matching qualitatively the original SIRUV model, just with slow parameters $\alpha$ and $\beta$ for the human dynamics. The two dimensional dynamical system then, by taking the conservations for human population $N=S+I$ and for mosquitoes $M=U+V$ into account, is given as

$$
\begin{aligned}
\frac{d}{d t} I & =\frac{\beta}{M}(N-I) V-\alpha I \\
\frac{d}{d t} V & =\frac{\vartheta}{N}(M-V) I-\nu V .
\end{aligned}
$$

The time scale separation is now given by the rescaling of the slow parameters $\beta=\varepsilon \bar{\beta}$ and $\alpha=\varepsilon \bar{\alpha}$. The endemic stationary state is given by

$$
I^{*}=\frac{\beta-\alpha \frac{\nu}{\vartheta}}{\beta+\alpha} \cdot N
$$

and as before

$$
V^{*}=\frac{\frac{\vartheta}{\nu} I^{*}}{N+\frac{\vartheta}{\nu} I^{*}} \cdot M
$$

\section{B.1 Linear part of the center manifold analysis for the SISUV model}

Then the Jacobian matrix around the endemic stationary state is given by

$$
\begin{aligned}
A & =\left.\left(\begin{array}{cc}
\frac{\partial f(I, V)}{\partial I} & \frac{\partial f(I, V)}{\partial V} \\
\frac{\partial g(I, V)}{\partial I} & \frac{\partial g(I, V)}{\partial V}
\end{array}\right)\right|_{\left(I^{*}, V^{*}\right)} \\
& =\left(\begin{array}{cc}
-\varepsilon \frac{\bar{\beta}}{M} \cdot V^{*}-\varepsilon \bar{\alpha} & \varepsilon \overline{\bar{\beta}} \cdot\left(N-I^{*}\right) \\
\frac{\vartheta}{N} \cdot\left(M-V^{*}\right) & -\frac{\vartheta}{N} I^{*}-\nu
\end{array}\right)=:\left(\begin{array}{ll}
a_{11} & a_{12} \\
a_{21} & a_{22}
\end{array}\right)
\end{aligned}
$$

or in scaling with $\varepsilon$

$$
A=\left(\begin{array}{cc}
\varepsilon \bar{a}_{11} & \varepsilon \bar{a}_{12} \\
a_{21} & a_{22}
\end{array}\right)
$$


The eigenvalues are given by

$$
\lambda_{1 / 2}=\frac{1}{2}\left(a_{22}+\varepsilon \bar{a}_{11}\right) \pm \sqrt{\frac{1}{4}\left(a_{22}+\varepsilon \bar{a}_{11}\right)^{2}-\varepsilon\left(\bar{a}_{11} a_{22}-\bar{a}_{12} a_{21}\right)}
$$

hence in leading order $\mathcal{O}\left(\varepsilon^{0}\right)$ we have

$$
\lambda_{1 / 2}=\frac{1}{2} a_{22} \pm \sqrt{\frac{1}{4} a_{22}^{2}}=\left\{\begin{array}{c}
0 \\
a_{22}
\end{array}\right.
$$

since $a_{22}$ is negative and hence $\sqrt{a_{22}^{2}}=-a_{22}$. This result was used previously in the center manifold analysis of the SISUV model [1]. Now in next to leading order we have the following result, by using

$$
f(\varepsilon):=(a+\varepsilon \cdot b)^{\frac{1}{2}}=f(0)+\left.\frac{d f}{d \varepsilon}\right|_{\varepsilon=0} \cdot \varepsilon+\mathcal{O}\left(\varepsilon^{2}\right)=\sqrt{a}+\frac{1}{2} \frac{b}{\sqrt{a}} \cdot \varepsilon+\mathcal{O}\left(\varepsilon^{2}\right)
$$

giving

$$
\begin{aligned}
& \lambda_{1}=\varepsilon\left(\bar{a}_{11}-\frac{\bar{a}_{12} a_{21}}{a_{22}}\right)+\mathcal{O}\left(\varepsilon^{2}\right) \\
& \lambda_{2}=a_{22}+\varepsilon \frac{\bar{a}_{12} a_{21}}{a_{22}}+\mathcal{O}\left(\varepsilon^{2}\right)
\end{aligned}
$$

hence

$$
\begin{aligned}
\lambda_{1} & =\varepsilon \bar{\lambda}_{1} \\
\lambda_{2} & =a_{22}+\varepsilon \bar{\lambda}_{2}
\end{aligned}
$$

with $\bar{\lambda}_{1}:=\bar{a}_{11}-\frac{\bar{a}_{12} a_{21}}{a_{22}}$ and $\bar{\lambda}_{2}:=\frac{\bar{a}_{12} a_{21}}{a_{22}}$.

Then the eigenvectors can be given as

$$
\underline{u}_{1}=\frac{1}{k_{1}}\left(\begin{array}{c}
\lambda_{1}-a_{22} \\
a_{21}
\end{array}\right)=\frac{1}{k_{1}}\left(\begin{array}{c}
-a_{22}+\varepsilon \bar{\lambda}_{1} \\
a_{21}
\end{array}\right)
$$

with eventually normalization constant $k_{1}=\sqrt{a_{21}^{2}+\left(-a_{22}+\varepsilon \bar{\lambda}_{1}\right)^{2}}$ and

$$
\underline{u}_{2}=\frac{1}{k_{2}}\left(\begin{array}{c}
\frac{\lambda_{2}-a_{22}}{a_{21}} \\
1
\end{array}\right)=\frac{1}{k_{2}}\left(\begin{array}{c}
\varepsilon \frac{\bar{\lambda}_{2}}{a_{21}} \\
1
\end{array}\right)
$$

with eventually normalization constant $k_{2}=\sqrt{1+\varepsilon^{2}\left(\bar{\lambda}_{2} / a_{21}\right)^{2}}=1+\mathcal{O}\left(\varepsilon^{2}\right)$, see footnote ${ }^{1}$. And with this we obtain the transformation matrix $T$ and its inverse $T^{-1}$ via $A T=T \Lambda$ with $A$ the Jacobian matrix as given above, and the matrix $\Lambda$ the diagonal matrix of eigenvalues, with

$$
T=\left(\underline{u}_{1}, \underline{u}_{2}\right)=\left(\begin{array}{ll}
u_{11} & u_{12} \\
u_{21} & u_{22}
\end{array}\right)=\left(\begin{array}{cc}
\frac{1}{k_{1}}\left(\lambda_{1}-a_{22}\right) & \frac{1}{k_{2}} \frac{\lambda_{2}-a_{22}}{a_{21}} \\
\frac{1}{k_{1}} a_{21} & \frac{1}{k_{2}}
\end{array}\right)
$$

\footnotetext{
${ }^{1}$ As an example of such calculations, here $f(\varepsilon):=\sqrt{1+\varepsilon^{2} c}$ which gives in Taylor's expansion $f(\varepsilon)=f(0)+\left.\frac{1}{2}\left(1+\varepsilon^{2} c\right)^{-1 / 2} \cdot 2 \varepsilon c\right|_{\varepsilon=0} \cdot \varepsilon+\mathcal{O}\left(\mu^{2}\right)$, and hence $f(\varepsilon)=1+0 \cdot \varepsilon+\mathcal{O}\left(\mu^{2}\right)=$ $1+\mathcal{O}\left(\mu^{2}\right)$.
} 
or in scaling with $\varepsilon$

$$
T=\left(\begin{array}{cc}
\frac{1}{k_{1}}\left(-a_{22}+\varepsilon \bar{\lambda}_{1}\right) & \varepsilon \frac{\bar{\lambda}_{2}}{a_{21}} \\
\frac{1}{k_{1}} a_{21} & 1
\end{array}\right)+\mathcal{O}\left(\varepsilon^{2}\right)
$$

(where the terms proportional to $1 / k_{1}$ could be analyzed further in scaling, but eventually disturbing the normalization and the geometric interpretation, this could be checked later in the calculations). The inverse $T^{-1}$ is given by

$T^{-1}=\frac{1}{u_{11} u_{22}-u_{12} u_{21}}\left(\begin{array}{cc}u_{22} & -u_{12} \\ -u_{21} & u_{11}\end{array}\right)=\frac{k_{1} k_{2}}{\lambda_{1}-\lambda_{2}}\left(\begin{array}{cc}\frac{1}{k_{2}} & -\frac{1}{k_{2}} \frac{\lambda_{2}-a_{22}}{a_{21}} \\ -\frac{1}{k_{1}} a_{21} & \frac{1}{k_{1}\left(\lambda_{1}-a_{22}\right)}\end{array}\right)$

and in scaling

$$
T^{-1}=\frac{1}{-a_{22}+\varepsilon\left(\bar{\lambda}_{1}-\bar{\lambda}_{2}\right)}\left(\begin{array}{cc}
k_{1} & -\varepsilon k_{1} \frac{\bar{\lambda}_{2}}{a_{21}} \\
-a_{21} & -a_{22}+\varepsilon \bar{\lambda}_{1}
\end{array}\right)+\mathcal{O}\left(\varepsilon^{2}\right)
$$

\section{B.2 Nonlinear part of the center manifold analysis for the SISUV model}

With the distance of the dynamical system from the endemic fixed point $\left(I^{*}, V^{*}\right)$ given by

$$
\underline{z}=\left(\begin{array}{c}
z \\
w
\end{array}\right):=\left(\begin{array}{c}
I(t)-I^{*} \\
V(t)-V^{*}
\end{array}\right)
$$

we have the linearized dynamics via the Jacobian matrix $A$ given as

$$
\frac{d}{d t} \underline{z}=A \underline{z}+\mathcal{O}\left(\|\underline{z}\|^{2}\right)
$$

and with the eigenvalue/eigenvector analysis $A T=T \Lambda$ the diagonalization of the dynamics

$$
\frac{d}{d t} \underline{x}=\Lambda \underline{x}+\mathcal{O}\left(\|\underline{x}\|^{2}\right)
$$

with the transformation

$$
\underline{z}=T \underline{x} \text {. }
$$

Now we take the nonlinear part of the dynamics $\underline{q}$ explicitly into account via

$$
\frac{d}{d t} \underline{z}=A \underline{z}+\underline{q}(\underline{z})
$$

with

$$
\underline{q}(\underline{z})=\left(\begin{array}{c}
-\frac{\beta}{M} \\
-\frac{\vartheta}{N}
\end{array}\right) \cdot(z \cdot w)
$$

and with the transformation $\underline{z}=T \underline{x}$ for $\underline{x}=:(x, y)^{\operatorname{tr}}$ we obtain

$\underline{q}(\underline{z})=\left(\begin{array}{c}-\frac{\beta}{M} \\ -\frac{\vartheta}{N}\end{array}\right) \cdot(z(x, y) \cdot w(x, y))=\left(\begin{array}{l}q_{1} \\ q_{2}\end{array}\right) \cdot\left(u_{11} x+u_{12} y\right) \cdot\left(u_{21} x+u_{22} y\right)=\underline{q}(T \underline{x})$ 
hence

$$
\underline{q}(T \underline{x})=\left(\begin{array}{c}
q_{1} \\
q_{2}
\end{array}\right) \cdot\left(\begin{array}{llll}
u_{11} & u_{21} & x^{2}+\left(\begin{array}{lll}
u_{11} & u_{22}+u_{12} & u_{21}
\end{array}\right) x y+u_{12} u_{22} y^{2}
\end{array}\right)=\left(\begin{array}{l}
q_{1} \\
q_{2}
\end{array}\right) \cdot\left(q_{3} x^{2}+q_{4} x y+q_{5} y^{2}\right)
$$

with constants $q_{1}:=-\varepsilon(\bar{\beta} / M), q_{2}:=-(\vartheta / N)$ and $q_{3}, q_{4}$ and $q_{5}$ given via

$$
\begin{aligned}
z(x, y) & =-\frac{a_{22}}{k_{1}} x+\varepsilon\left(\frac{\bar{\lambda}_{1}}{k_{1}} x+\frac{\bar{\lambda}_{2}}{a_{21}} y\right) \\
w(x, y) & =\frac{a_{21}}{k_{1}} x+y
\end{aligned}
$$

such that we have now the complete nonlinear dynamical system in transformed variables for the diagonalized linear part as

$$
\frac{d}{d t} \underline{x}=\Lambda \underline{x}+T^{-1} \underline{q}(T \underline{x})
$$

with the nonlinear part given by

$$
T^{-1} \underline{q}(T \underline{x})=\frac{1}{u_{11} u_{22}-u_{12} u_{21}}\left(\begin{array}{cc}
u_{22} & -u_{12} \\
-u_{21} & u_{11}
\end{array}\right) \cdot\left(\begin{array}{c}
q_{1} \\
q_{2}
\end{array}\right) z(x, y) \cdot w(x, y)
$$

and abbreviating

$$
T^{-1} \underline{q}(T \underline{x})=:\left(\begin{array}{c}
f_{c} \\
g_{c}
\end{array}\right) z(x, y) \cdot w(x, y)=:\left(\begin{array}{c}
f_{n l}(x, y) \\
g_{n l}(x, y)
\end{array}\right)
$$

with constants

$$
f_{c}:=\frac{q_{1} u_{22}-q_{2} u_{12}}{u_{11} u_{22}-u_{12} u_{21}}
$$

and

$$
g_{c}:=\frac{-q_{1} u_{21}+q_{2} u_{11}}{u_{11} u_{22}-u_{12} u_{21}}
$$

giving explicitly and in scaling

$$
f_{c}=\frac{-\frac{\beta}{M} \frac{1}{k_{2}} \frac{\vartheta}{N}\left(-\frac{1}{k_{2}} \frac{\lambda_{2}-a_{22}}{a_{21}}\right)}{\lambda_{1}-\lambda_{2}}=\varepsilon k_{1} \frac{-\frac{\bar{\beta}}{M}+\frac{\vartheta}{N} \frac{\bar{\lambda}_{2}}{a_{21}}}{-a_{22}+\varepsilon\left(\bar{\lambda}_{1}-\bar{\lambda}_{2}\right)}+\mathcal{O}\left(\varepsilon^{2}\right)
$$

and

$$
g_{c}=\frac{-\frac{\beta}{M} \frac{a_{21}}{k_{1}} \frac{\vartheta}{N} \frac{1}{k_{1}}\left(\lambda_{2}-a_{22}\right)}{\lambda_{1}-\lambda_{2}}=\frac{\frac{\vartheta}{N}\left(a_{22}-\varepsilon \bar{\lambda}_{1}\right)-\varepsilon \frac{\bar{\beta}}{M} a_{21}}{-a_{22}+\varepsilon\left(\bar{\lambda}_{1}-\bar{\lambda}_{2}\right)}+\mathcal{O}\left(\varepsilon^{2}\right)
$$

Hence we have the dynamical system now as

$$
\begin{aligned}
& \frac{d}{d t} x=\lambda_{1} x+f_{n l}(x, y) \\
& \frac{d}{d t} y=\lambda_{2} y+g_{n l}(x, y)
\end{aligned}
$$


and assuming the fast variable $y$ as a function of the slow variable $x$, hence $y=h(x)$, we have by using the chain rule of differentiation

$$
\begin{aligned}
\frac{d}{d t} y & =\lambda_{2} h(x)+g_{n l}(x, h(x)) \\
& =\frac{d h(x)}{d x} \cdot \frac{d x}{d t} \\
& =\frac{d h(x)}{d x} \cdot\left(\lambda_{1} x+f_{n l}(x, h(x))\right)
\end{aligned}
$$

This gives the functional $\mathcal{N}(h(x))$ of the unknown function $h(x)$ as a functional equation $\mathcal{N}(h(x))=0$ to be solved with

$$
0=\mathcal{N}(h(x))=\frac{d h(x)}{d x} \cdot\left(\lambda_{1} x+f_{n l}(x, h(x))\right)-\left(\lambda_{2} h(x)+g_{n l}(x, h(x))\right)
$$

This could be done with a polynomial ansatz for $h(x):=h_{2} x^{2}+h_{3} x^{3}+h_{4} x^{4}+$ $\mathcal{O}\left(x^{5}\right)$ around the center, hence small $x$ [1], giving recursions for higher orders of $h_{\nu}$ as functions of lower, similar to what is reported in [8].

But given the analysis of all terms in scaling with the small parameter $\varepsilon$, we find that as well $\lambda_{1}=\mathcal{O}(\varepsilon)$ as $f_{n l}(x, h(x))=\mathcal{O}(\varepsilon)$ due to $f_{c}=\mathcal{O}(\varepsilon)$. Hence in leading order $\mathcal{O}(1)$ we have from Eq. (141) only an algebraic equation left

$$
0=\lambda_{2} h(x)+g_{n l}(x, h(x))
$$

So we can in this case use the same technique as in singular perturbation, namely we expand $h$ in powers of the small scaling parameter $\varepsilon$ with

$$
h(x, \varepsilon)=h_{0}(x)+h_{1}(x) \cdot \varepsilon+\mathcal{O}\left(\varepsilon^{2}\right)
$$

such that Eq. (141) can be solved successively in increasing orders of $\varepsilon$, starting with lowest order

$$
0=\lambda_{2} h_{0}(x)+g_{n l}\left(x, h_{0}(x)\right)
$$

which can be solved directly giving

$$
\lambda_{2} \cdot y(w, z)-\frac{\vartheta}{N} z w=0
$$

or

$$
w=\frac{-a_{21} z}{a_{22}-\frac{\vartheta}{N} z}
$$

and with $z=V(t)-V^{*}$ and $w=I(t)-I^{*}$ we obtain

$$
V_{0}(I)=\frac{\frac{\vartheta}{\nu} I(t)}{N+\frac{\vartheta}{\nu} I(t)} \cdot M
$$

analogously to the result from singular perturbation via the ansatz $V(I, \varepsilon)=$ $V_{0}(I)+V_{1}(I) \cdot \varepsilon+\mathcal{O}\left(\varepsilon^{2}\right)$ directly applied to Eq. (107) and with the scaling $\beta=\varepsilon \bar{\beta}$ and $\alpha=\varepsilon \bar{\alpha}$.

And then as in singular perturbation the next order terms can be calculated using the lowest order result to calculate the next order via Eq. (141). In this 
respect center manifold analysis gives the same result to all orders as singular perturbation in the cases where $\frac{d h(x)}{d x} \cdot\left(\lambda_{1} x+f_{n l}(x, h(x))\right)$ is of higher order than $\lambda_{2} h(x)+g_{n l}(x, h(x))$, i.e. the standard time scale separation form $d x / d t=\varepsilon f$ and $d y / d t=g$.

Just in cases not in standard time scale separation form, the Eq. (141) can be solved via $h$ expanding in small scaling parameter $\varepsilon$ and in slow state variable $x$, and then goes beyond singular perturbation (as we see in the SIRUV system in the main text). Else, singular perturbation gives already the answer of the slow mainfold $y=h(x, \varepsilon)$, and center manifold analysis only adds the linear algebra part of eigenvalues and eigendirections. 


\section{Transition from slow-fast time scale separation to final spiraling on center manifold}

From the scaling of the state variables at the endemic fixed point, Eqs. (88), (89) and (90), we have $I^{*}=\mathcal{O}(\mu)$ and with it also $V^{*}=\mathcal{O}(\mu)$, whereas $S^{*}=\mathcal{O}(1)$. We can apply this as scaling transformations for the state variables outside the endemic equilibrium, hence

$$
\begin{array}{rll}
I(t) & =: & \mu \cdot \bar{I}(t) \\
V(t) & =: & \mu \cdot \bar{V}(t)
\end{array}
$$

with $\bar{I}(t)$ and $\bar{V}(t)$ in roughly the magnitude of $S^{*}$. By inserting this transformation into the dynamic equations, Eq. (2), we obtain for the variables $S(t)$, $\bar{I}(t)$ and $\bar{V}(t)$ the dynamical system

$$
\begin{aligned}
\frac{d}{d t} S & =\mu\left((N-S)-\frac{\beta}{M} S \bar{V}\right) \\
\frac{d}{d t} \bar{I} & =\frac{\beta}{M} S \bar{V}-(\gamma+\mu) \bar{I} \\
\frac{d}{d t} \bar{V} & =\frac{\vartheta}{N}(M-\mu \cdot \bar{V}) \bar{I}-\nu \bar{V}
\end{aligned}
$$

which has now in the small parameter $\mu$ one slow variable, namely $S$, and two fast variables, $\bar{V}$ and $\bar{I}$.

In this form with variables $S, \bar{V}$ and $\bar{I}$ we have exactly the standard singular perturbation form given by

$$
\begin{aligned}
\frac{d}{d t} S & =\mu \cdot f(S, \bar{I}, \bar{V}, \mu) \\
\frac{d}{d t}\left(\begin{array}{c}
\bar{I} \\
\bar{V}
\end{array}\right) & =\underline{g}(S, \bar{I}, \bar{V}, \mu)
\end{aligned}
$$

with one slow and two fast variable. This, of course, cannot be the dynamic regime close to the endemic fixed point, since there we have two slow variables $S$ and $I$ and one fast variable $V$, but rather describes a time scale separable regime in the transient phase, where we observe from a state of low numbers of susceptibles a slow building-up phase of susceptibles $S$ with very low numbers of infected humans $I$ and infected mosquitoes $V$, hence $I^{*}=\mathcal{O}(\mu)$ and $V^{*}=\mathcal{O}(\mu)$, and then in a state with many susceptibles a fast burst of infecteds, infected humans $I$ and infected mosquitoes $V$, until they burn out more susceptibles than would be needed to keep a high infection level. This fast bursting phase is then followed again by a slow rebuilding of susceptibles and again in low numbers of infected, $I$ and $V$.

The slow-fast build-up and burst dynamics can be observed in the SIRUV model, as it can be in other SIR-type models (see [12] for such slow-fast dynamics in some SIR-type models), when starting the dynamics with initial conditions far away from the endemic fixed point, see Fig. 3. In a) we observe from 
a)

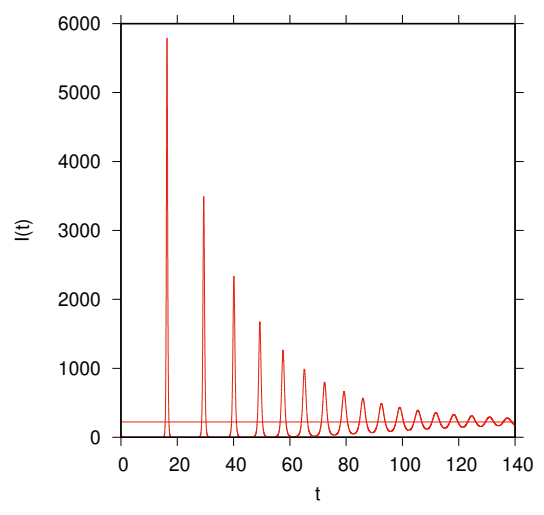

b)

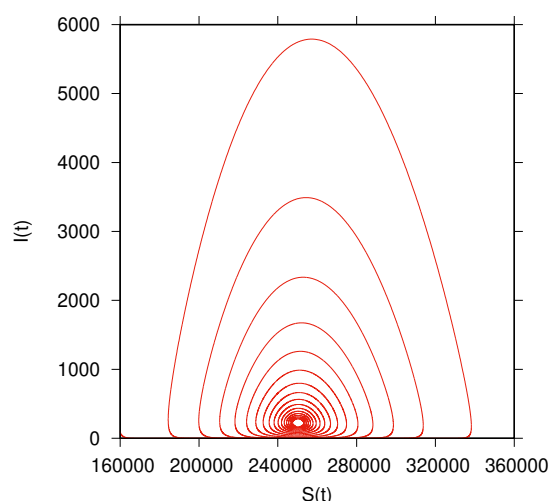

$\mathrm{S}(\mathrm{t})$

Figure 3: Time series of infected and state space plot of infected versus susceptibles in the SIRUV model. Initial conditions far away from the endemic fixed point, $S_{0}=0.17 \cdot N, I_{0}=0.0001 \cdot N$, and small number or no infected mosquitoes. After long times with low infection rate and slow build up of susceptibles we observe fast large outbreaks of infected, burning out large proportions of susceptibles (slow-fast regime). Then we observe a gradual transition to oscillations into the endemic fixed point with the known dynamics in the vicinity of the fixed point described (center manifold regime).

initially very low numbers of infected a sudden spike of number of infected (just before $t=20$ ), followed by another phase of very low numbers of infected, in which the susceptibles are built up again, followed by another spiking. This is the slow-fast dynamics phase. However, gradually the spikes decrease and the troughs in the low infection phases increase, until the dynamics finally enters into the oscillatory phase around the endemic fixed point, the regime which is described by the time scale separation towards the center manifold. The relation between bursting infected and susceptibles re-building becomes more obvious in the state space plot in Fig. 3 b), where from about 160000 susceptibles and very low numbers of infected the susceptibles reach a level of about 340 000, from which on the number of infected rapidly increases, before crashing again at numbers of susceptibles around 180000 etc. Finally, we can also observe the spiraling into the endemic fixed point at around 250000 susceptibles, similar to what was observe in Fig. 1 b), there with initial conditions close to the endemic fixed point. 


\section{Quadratic approximation of center manifold}

From

$$
z_{3}=z_{3}\left(z_{2}, z_{1}\right)=x_{2}+y
$$

and

$$
y=h\left(z_{2}, z_{1}\right)=h_{02} \cdot x_{2}^{2}+h_{11} \cdot x_{1} x_{2}+\mathcal{O}\left(\|\underline{x}\|^{3}\right)
$$

we obtain with the transformations from the center manifold coordinates $\underline{x}$ to the original coordinates $\underline{z}$, remembering that $z_{1}:=S(t)-S^{*}, z_{2}:=I(t)-I^{*}$ and $z_{3}:=V(t)-V^{*}$, with

$$
x_{1}=z_{1} \quad, \quad x_{2}=\frac{1}{\varphi-\psi}\left(z_{2}-\psi z_{3}\right)
$$

the expression

$$
\begin{aligned}
z_{3} & =h_{02} \cdot x_{2}^{2}+\left(1+h_{11} \cdot x_{1}\right) \cdot x_{2} \\
& =h_{02}\left(\frac{1}{\varphi-\psi}\right)^{2}\left(z_{2}-\psi z_{3}\right)^{2}+\left(1+h_{11} \cdot z_{1}\right) \cdot \frac{1}{\varphi-\psi}\left(z_{2}-\psi z_{3}\right)
\end{aligned}
$$

which has to be solved for $z_{3}=z_{3}\left(z_{2}, z_{1}\right)$.

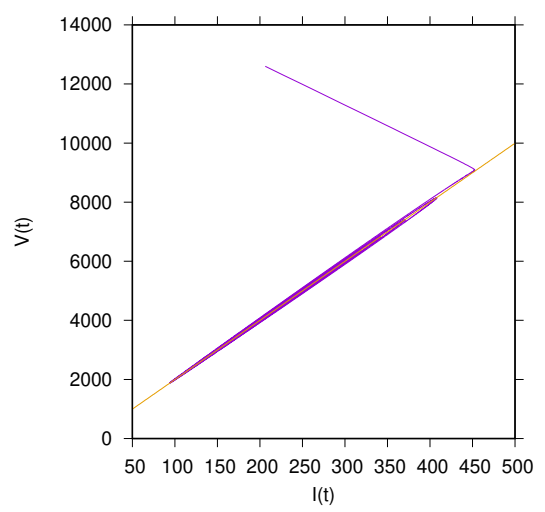

Figure 4: Quadratic approximation of $z_{3}=z_{3}\left(z_{2}, z_{1}\right)$ for continuously varying $z_{2}$ and $z_{1}=0$ in comparison with the trajectory spiralling into the fixed point. The quadratic expression gives essentially the linear relation between $V$ and $I$ again, namely $V(I)=\frac{\vartheta}{\nu} \frac{M}{N} I(t)$.

The quadratic equation for $z_{3}$ is given by

$$
\begin{aligned}
z_{3}^{2} & -\left(\frac{2}{\psi} z_{2}+\left(\frac{\varphi-\psi}{\psi}\right)^{2} \cdot \frac{1}{h_{02}}\left(1+\frac{\psi}{\varphi-\psi}\left(1+h_{11} \cdot z_{1}\right)\right)\right) \cdot z_{3} \\
& +\frac{1}{\psi^{2}} z_{2}^{2}+\frac{\varphi-\psi}{\psi^{2}} \cdot \frac{1}{h_{02}}\left(1+h_{11} \cdot z_{1}\right) \cdot z_{2} \\
& =0
\end{aligned}
$$

hence with

$$
z_{3}^{2}+p\left(z_{2}, z_{1}\right) \cdot z_{3}+q\left(z_{2}, z_{1}\right)=0
$$


the solution $z_{3}\left(z_{2}, z_{1}\right)$ is given by

$$
z_{3}=-\frac{p}{2}+\sqrt{\left(\frac{p}{2}\right)^{2}-q} \approx \frac{1}{\varphi} \cdot z_{2}=\frac{\vartheta}{\nu} \frac{M}{N} \cdot z_{2}
$$

and plotted graphically for $z_{1}=0$ in Fig. 4. The coefficients for the quadratic approximation of the center manifold $z=h\left(x_{1}, x_{2}\right)$ are $h_{11}=-\frac{1}{\gamma+\nu} \cdot \frac{1}{\varphi-\psi} \frac{\beta}{M}$, giving in original model parameters $h_{11}=-\frac{\vartheta \beta}{(\gamma+\nu)^{2}} \cdot \frac{1}{N}$, and furthermore the second coefficient $h_{02}=-\frac{1}{\gamma+\nu}\left(h_{11} \xi+\frac{\varphi^{2}}{\varphi-\psi} \frac{\vartheta}{N}\right)$, which is in original model parameters $h_{02}=-\left(\frac{\nu}{\gamma+\nu}\right)^{2}\left(1+\frac{\gamma}{\nu} \cdot \frac{\beta}{\gamma+\nu}\right) \cdot \frac{1}{M}$.

a)

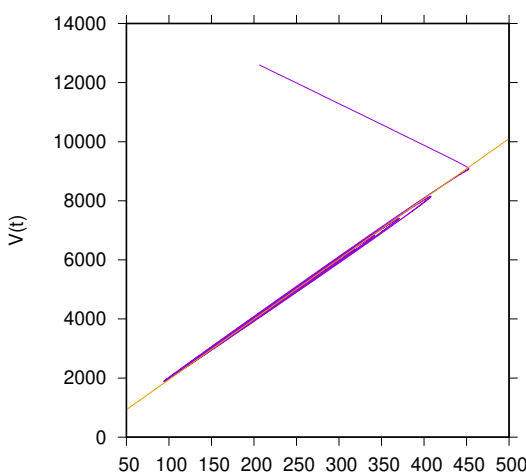

I(t)

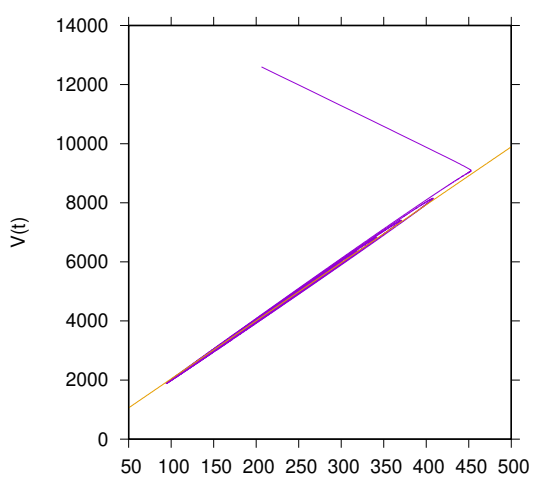

b)

Figure 5: Quadratic approximation of $z_{3}=z_{3}\left(z_{2}, z_{1}\right)$ for continuously varying $z_{2}$ and different values of $z_{1}$, a) $z_{1}=242000-S^{*}$ and b) $z_{1}=258000-S^{*}$, values in the range of the oscillations of $S(t)$ into the fixed point.

In Fig. 5 we explore the $z_{1}$ dependence of $z_{3}=z_{3}\left(z_{2}, z_{1}\right)$ further by comparing the graphs for $z_{1}=242000-S^{*}$, the smallest value of $S$ when spiralling into the fixed point and $z_{1}=258000-S^{*}$, the largest value of $S$ when spiralling into the fixed point. The slight variations of the lines for the varying $z_{1}$ values around the value $z_{1}=0$, the mid point, see. Fig. 4, are in the range of the variations of the oscillating trajectory. 


\section{E Quadratic approximation of center manifold in next to leading order in $\mu$}

\section{E.1 Analysis of determining equation of $w_{1}$}

For the family of center manifolds

$$
\begin{aligned}
& \frac{d}{d t} \underline{x}=\underline{P}(\underline{x}, y, \mu) \\
& \frac{d}{d t} y=Q(\underline{x}, y, \mu)
\end{aligned}
$$

we have for $y=w(\underline{x}, \mu)$ the detrmining equation for $w(\underline{x}, \mu)$ given as

$$
\frac{d w}{d \underline{x}} \underline{P}-Q=0
$$

and expand in orders of $\mu$ in the following way

$$
\begin{aligned}
y=w(\underline{x}, \mu) & =w_{0}(\underline{x})+\mu w_{1}(\underline{x})+\mathcal{O}\left(\mu^{2}\right) \\
\underline{P}(\underline{x}, y, \mu) & =\underline{P}_{0}(\underline{x}, y)+\mu \underline{P}_{1}(\underline{x}, y)+\mathcal{O}\left(\mu^{2}\right) \\
Q(\underline{x}, y, \mu) & =Q_{0}(\underline{x}, y)+\mu Q_{1}(\underline{x}, y)+\mathcal{O}\left(\mu^{2}\right)
\end{aligned}
$$

taking care of all variable-dependences, and remembering from zeroth order in $\mu$ that $w_{0}(\underline{x})=h(\underline{x})$. Hence in next to leading order in $\mu$ we have

$$
\frac{d\left(w_{0}+\mu w_{1}\right)}{d \underline{x}}\left(\underline{P}_{0}+\mu \underline{P}_{1}\right)-\left(Q_{0}+\mu Q_{1}\right)=0
$$

or

$$
\underbrace{\left(\frac{d h}{d \underline{x}} \underline{P}_{0}-Q_{0}\right)}_{=: D}+\mu \cdot\left(\frac{d h}{d \underline{x}} \underline{P}_{1}+\frac{d w_{1}}{d \underline{x}} \underline{P}_{0}-Q_{1}\right)=0
$$

where we still have to evaluate $\underline{P}_{i}(\underline{x}, y)$ and $Q_{i}(\underline{x}, y)$ with $y=h(\underline{x})+\mu w_{1}(\underline{x})$. It is explicitly

$$
\begin{aligned}
Q_{i}(\underline{x}, y) & =Q_{i}\left(\underline{x}, h(\underline{x})+\mu w_{1}(\underline{x})\right) \\
& =Q_{i}(\underline{x}, h(\underline{x}))+\left.\frac{\partial}{\partial y} Q_{i}(\underline{x}, y)\right|_{y=h(\underline{x})} \cdot \mu w_{1}(\underline{x})+\mathcal{O}\left(\mu^{2}\right)
\end{aligned}
$$

and respectively for $\underline{P}_{i}(\underline{x}, y)$. This gives for $D:=\frac{d h}{d \underline{x}} \underline{P}_{0}-Q_{0}$ the result to first order in $\mu$ as

$$
\begin{aligned}
D= & \underbrace{\left(\frac{d h}{d \underline{x}} \cdot \underline{P}_{0}(\underline{x}, h(\underline{x}))-Q_{0}(\underline{x}, h(\underline{x}))\right)}_{=0} \\
& +\left.\frac{d h}{d \underline{x}} \cdot \frac{\partial}{\partial y} \underline{P}_{0}(\underline{x}, y)\right|_{y=h(\underline{x})} \cdot \mu w_{1}(\underline{x})+\left.\frac{\partial}{\partial y} Q_{0}(\underline{x}, y)\right|_{y=h(\underline{x})} \cdot \mu w_{1}(\underline{x})+\mathcal{O}\left(\mu^{2}\right) \\
= & D_{0}+\mu D_{1}+\mathcal{O}\left(\mu^{2}\right)
\end{aligned}
$$


with $D_{0}=0$ from the zeroth order calculation in $\mu$ by determining $h(\underline{x})$. Further considering terms like

$$
\mu Q_{1}(\underline{x}, y)=\mu Q_{1}(\underline{x}, h)+\left.\mu^{2} \cdot \frac{\partial}{\partial y} Q_{0}(\underline{x}, y)\right|_{y=h(\underline{x})} \cdot w_{1}(\underline{x})+\mathcal{O}\left(\mu^{2}\right)
$$

we obtain in $\mathcal{O}(\mu)$ the determining equation for $w_{1}(\underline{x})$ as

$$
\frac{d h}{d \underline{x}} \underline{P}_{1}(\underline{x}, h(\underline{x}))+\frac{d w_{1}}{d \underline{x}} \underline{P}_{0}(\underline{x}, h(\underline{x}))-Q_{1}(\underline{x}, h(\underline{x}))+D_{1}=0
$$

with

$$
D_{1}=\left.\frac{d h}{d \underline{x}} \cdot \frac{\partial}{\partial y} \underline{P}_{0}(\underline{x}, y)\right|_{y=h(\underline{x})} \cdot w_{1}(\underline{x})+\left.\frac{\partial}{\partial y} Q_{0}(\underline{x}, y)\right|_{y=h(\underline{x})} \cdot w_{1}(\underline{x})
$$

and hence finally

$\frac{d h}{d \underline{x}}\left(\underline{P}_{1}(\underline{x}, h(\underline{x}))+\left.\frac{\partial}{\partial y} \underline{P}_{0}(\underline{x}, y)\right|_{y=h(\underline{x})} \cdot w_{1}(\underline{x})\right)+\frac{d w_{1}}{d \underline{x}} \underline{P}_{0}(\underline{x}, h(\underline{x}))-\left(Q_{1}(\underline{x}, h(\underline{x}))+\left.\frac{\partial}{\partial y} Q_{0}(\underline{x}, y)\right|_{y=h(\underline{x})} \cdot w_{1}(\underline{x})\right)=0$

taking care of all variable dependences, or in short hand notation

$$
\frac{d h}{d \underline{x}}\left(\underline{P}_{1}+\left.\frac{\partial}{\partial y} \underline{P}_{0}\right|_{y=h} \cdot w_{1}\right)+\frac{d w_{1}}{d \underline{x}} \underline{P}_{0}-\left(Q_{1}+\left.\frac{\partial}{\partial y} Q_{0}\right|_{y=h} \cdot w_{1}\right)=0
$$

From this equation we now can calculate $w_{1}(\underline{x})$ in quadratic order, since we already have $h(\underline{x})=w_{0}(\underline{x})$ in this quadratic order, with the ansatz for $w_{1}(\underline{x})$ in the form

$$
w_{1}(\underline{x})=w_{1,1} x_{1}+w_{2,1} x_{2}+w_{20,1} x_{1}^{2}+w_{11,1} x_{1} x_{2}+w_{02,1} x_{2}^{2}+\mathcal{O}\left(\|\underline{x}\|^{3}\right)
$$

\section{E.2 Calculation of functions $\underline{P}_{0}, Q_{0}, \underline{P}_{1}$, etc.}

We now calculate the functions $\underline{P}_{0}(\underline{x}, y), Q_{0}(\underline{x}, y), \underline{P}_{1}(\underline{x}, y), Q_{1}(\underline{x}, y)$ and the derivatives $\frac{\partial}{\partial y} \underline{P}_{0}(\underline{x}, y)$ and $\frac{\partial}{\partial y} Q_{0}(\underline{x}, y)$ to order $\mathcal{O}\left(\|\underline{x}\|^{2}\right)$, remembering that $y=$ $h(\underline{x})$ to be inserted is of quadratic order, in a form that we can then easily access zeroth order, linear and quadratic terms in $\underline{x}$.

First we calculate $\underline{P}_{0}$ and $Q_{0}$ such that we then can easily take the derivatives in respect to $y$. We have from

$$
\begin{aligned}
\frac{d}{d t} \underline{z} & =A \underline{z}+\underline{q}(\underline{z})=A_{0} \underline{z}+\underline{q}(\underline{z})+\mathcal{O}\left(\mu^{2}\right) \\
& =A_{0} \underline{z}+\left(\begin{array}{c}
-\frac{\beta}{M} z_{1} \\
\frac{\beta}{M} z_{1} \\
\frac{\vartheta}{M} z_{2}
\end{array}\right) \cdot z_{3}+\mathcal{O}\left(\mu^{2}\right) \\
& =A_{0} \underline{z}+\left(\begin{array}{ccc}
-\frac{\beta}{M} & 0 & 0 \\
\frac{\beta}{M} & 0 & 0 \\
0 & \frac{\vartheta}{M} & 0
\end{array}\right)\left(\begin{array}{l}
z_{1} \\
z_{2} \\
z_{3}
\end{array}\right) \cdot z_{3}+\mathcal{O}\left(\mu^{2}\right)
\end{aligned}
$$


with the matrix

$$
\mathcal{N}:=\left(\begin{array}{ccc}
-\frac{\beta}{M} & 0 & 0 \\
\frac{\beta}{M} & 0 & 0 \\
0 & \frac{\vartheta}{M} & 0
\end{array}\right)
$$

in coordinates $\underline{x}_{3}=\tilde{T}^{-1} \underline{z}=\left(x_{1}, x_{2}, y\right)^{t r}$

$$
\begin{aligned}
\frac{d}{d t} \underline{x}_{3} & =\tilde{T}^{-1} A_{0} \tilde{T} \underline{x}_{3}+\tilde{T}^{-1} \underline{q}\left(\tilde{T} \underline{x}_{3}\right)+\mathcal{O}(\mu) \\
& =\left(\begin{array}{l}
\underline{P}_{0}(\underline{x}, y) \\
Q_{0}(\underline{x}, y)
\end{array}\right)+\mathcal{O}(\mu)
\end{aligned}
$$

with $\underline{x}=\left(x_{1}, x_{2}\right)^{t r}$.

Hence with the explicit transformation $z_{3}=x_{2}+y$ we obtain the functions $\underline{P}_{0}(\underline{x}, y)$ and $Q_{0}(\underline{x}, y)$ via

$$
\begin{aligned}
\left(\begin{array}{c}
\underline{P}_{0}(\underline{x}, y) \\
Q_{0}(\underline{x}, y)
\end{array}\right) & =\tilde{T}^{-1} A_{0} \tilde{T} \underline{x}_{3}+\tilde{T}^{-1} \underline{q}\left(\tilde{T} \underline{x}_{3}\right) \\
& =\tilde{T}^{-1} A_{0} \tilde{T} \underline{x}_{3}+\tilde{T}^{-1} \mathcal{N} \tilde{T} \underline{x}_{3} \cdot\left(x_{2}+y\right) \\
& =\tilde{T}^{-1} A_{0} \tilde{T}\left(\begin{array}{c}
x_{1} \\
x_{2} \\
y
\end{array}\right)+\tilde{T}^{-1} \mathcal{N} \tilde{T}\left(\begin{array}{c}
x_{1} \\
x_{2} \\
y
\end{array}\right) \cdot\left(x_{2}+y\right)
\end{aligned}
$$

with the matrices

$$
\tilde{T}^{-1} A_{0} \tilde{T}=\left(\begin{array}{ccc}
0 & \xi & \xi \\
0 & 0 & 0 \\
0 & 0 & -(\gamma+\nu)
\end{array}\right)
$$

and

$$
\begin{aligned}
\mathcal{M} & :=\tilde{T}^{-1} \mathcal{N} \tilde{T} \\
& =\frac{1}{\gamma+\nu} \cdot \frac{1}{M}\left(\begin{array}{ccc}
-\beta(\gamma-\nu) & 0 & 0 \\
-\vartheta \beta \frac{M}{N} & \nu \gamma & -\gamma^{2} \\
\vartheta \beta \frac{M}{N} & \nu^{2} & -\nu \gamma
\end{array}\right)=:\left(\begin{array}{ccc}
m_{11} & 0 & 0 \\
-m_{31} & m_{22} & m_{23} \\
m_{31} & m_{32} & m_{33}
\end{array}\right)
\end{aligned}
$$

Hence we obtain for $\underline{P}_{0}(\underline{x}, y)$ explicitly

$$
\begin{aligned}
\underline{P}_{0}(\underline{x}, y) & =\left(\begin{array}{c}
\xi \\
0
\end{array}\right)\left(x_{2}+y\right)+\left(\begin{array}{ccc}
m_{11} & 0 & 0 \\
-m_{31} & m_{22} & m_{23}
\end{array}\right)\left(\begin{array}{c}
x_{1} \\
x_{2} \\
y
\end{array}\right) \cdot\left(x_{2}+y\right) \\
& =\left[\left(\begin{array}{l}
\xi \\
0
\end{array}\right)+\left(\begin{array}{cc}
m_{11} & 0 \\
-m_{31} & m_{22}
\end{array}\right) \cdot\left(\begin{array}{l}
x_{1} \\
x_{2}
\end{array}\right)+\left(\begin{array}{c}
0 \\
m_{23}
\end{array}\right) \cdot y\right] \cdot\left(x_{2}+y\right)
\end{aligned}
$$

and for $Q_{0}(\underline{x}, y)$ explicitly

$$
\begin{aligned}
Q_{0}(\underline{x}, y) & =-(\gamma+\nu) y+\left(m_{31}, m_{32}, m_{33}\right)\left(\begin{array}{c}
x_{1} \\
x_{2} \\
y
\end{array}\right) \cdot\left(x_{2}+y\right) \\
& =-(\gamma+\nu) y+\left[\left(m_{31}, m_{32}\right)\left(\begin{array}{c}
x_{1} \\
x_{2}
\end{array}\right)+m_{33} \cdot y\right] \cdot\left(x_{2}+y\right)
\end{aligned}
$$


and from these also the derivatives

$$
\frac{\partial}{\partial y} \underline{P}_{0}(\underline{x}, y)=\left(\begin{array}{c}
\xi \\
0
\end{array}\right)+\left(\begin{array}{cc}
m_{11} & 0 \\
-m_{31} & m_{22}+m_{23}
\end{array}\right) \cdot\left(\begin{array}{l}
x_{1} \\
x_{2}
\end{array}\right)+\left(\begin{array}{c}
0 \\
2 m_{23}
\end{array}\right) \cdot y
$$

and

$$
\frac{\partial}{\partial y} Q_{0}(\underline{x}, y)=-(\gamma+\nu)+\left(m_{31}, m_{32}+m_{33}\right)\left(\begin{array}{c}
x_{1} \\
x_{2}
\end{array}\right)+2 m_{33} \cdot y
$$

To determin $\underline{P}_{1}$ and $Q_{1}$ we have to take the expansion of $A=A_{0}+\mu A_{1}+\mathcal{O}\left(\mu^{2}\right)$ into account, since the nonlinear part $q(\underline{z})$ is $\mu$-independent in

$$
\begin{aligned}
\frac{d}{d t} \underline{x}_{3} & =\left(\begin{array}{c}
\underline{P}_{0}(\underline{x}, y) \\
Q_{0}(\underline{x}, y)
\end{array}\right)+\mu\left(\begin{array}{c}
\underline{P}_{1}(\underline{x}, y) \\
Q_{1}(\underline{x}, y)
\end{array}\right)+\mathcal{O}\left(\mu^{2}\right) \\
& =\left(\begin{array}{c}
\underline{P}_{0}(\underline{x}, y) \\
Q_{0}(\underline{x}, y)
\end{array}\right)+\mu \tilde{T}^{-1} A_{1} \tilde{T} \underline{x}_{3}+\mathcal{O}\left(\mu^{2}\right)
\end{aligned}
$$

and hence

$$
\begin{aligned}
& \left(\begin{array}{l}
\underline{P}_{1}(\underline{x}, y) \\
Q_{1}(\underline{x}, y)
\end{array}\right)=\tilde{T}^{-1} A_{1} \tilde{T} \underline{x}_{3}
\end{aligned}
$$

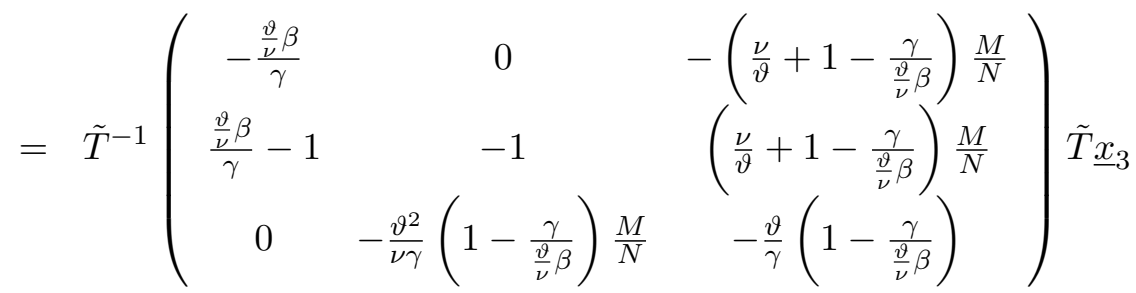

$$
\begin{aligned}
& =\tilde{T}^{-1}\left(\begin{array}{ccc}
-\alpha_{11} & 0 & -\alpha_{23} \\
\alpha_{11}-1 & -1 & \alpha_{23} \\
0 & -\alpha_{32} & -\alpha_{33}
\end{array}\right) \tilde{T} \underline{x}_{3} \\
& =\left(\begin{array}{ccc}
-\alpha_{11} & \alpha_{23} & -\alpha_{23} \\
\frac{\alpha_{11}-1}{\varphi-\psi} & \frac{\varphi\left(\psi \alpha_{32}-1\right)+\alpha_{23}+\psi \alpha_{33}}{\varphi-\psi} & \frac{\psi\left(\psi \alpha_{32}-1\right)+\alpha_{23}+\psi \alpha_{33}}{\varphi-\psi} \\
\frac{-\left(\alpha_{11}-1\right)}{\varphi-\psi} & \frac{-\left(\varphi\left(\varphi \alpha_{32}-1\right)+\alpha_{23}+\varphi \alpha_{33}\right)}{\varphi-\psi)} & \frac{-\left(\psi\left(\varphi \alpha_{32}-1\right)+\alpha_{23}+\varphi \alpha_{33}\right)}{\varphi-\psi)}
\end{array}\right) \cdot \underline{x}_{3} \\
& =\left(\begin{array}{llll}
r_{11} & r_{12} & r_{13} & y \\
r_{21} & r_{22} & r_{23} & y \\
r_{31} & r_{32} & r_{33} & y
\end{array}\right) \cdot\left(\begin{array}{c}
x_{1} \\
x_{2} \\
y
\end{array}\right) \\
& =\mathcal{R} \underline{x}_{3}
\end{aligned}
$$

such that we obtain $\underline{P}_{1}$ and $Q_{1}$ as

$$
\underline{P}_{1}(\underline{x}, y)=\left(\begin{array}{ll}
r_{11} & r_{12} \\
r_{21} & r_{22}
\end{array}\right)\left(\begin{array}{c}
x_{1} \\
x_{2}
\end{array}\right)+\left(\begin{array}{l}
r_{13} \\
r_{23}
\end{array}\right) y
$$

and

$$
Q_{1}(\underline{x}, y)=\left(r_{31}, r_{32}\right)\left(\begin{array}{l}
x_{1} \\
x_{2}
\end{array}\right)+r_{33} \cdot y
$$

with $r_{i j}$ the matrix entries of $\mathcal{R}$. 


\section{E.3 Calculation of coefficients of $w_{1}$ to second order in $\underline{x}$}

With the functions $\underline{P}_{0}, Q_{0}, \underline{P}_{1}, Q_{1}$ and $\frac{\partial}{\partial y} \underline{P}_{0}(\underline{x}, y), \frac{\partial}{\partial y} Q_{0}(\underline{x}, y)$ calculated as

$$
\begin{aligned}
& \underline{P}_{0}(\underline{x}, y)=\left[\left(\begin{array}{l}
\xi \\
0
\end{array}\right)+\left(\begin{array}{cc}
m_{11} & 0 \\
-m_{31} & m_{22}
\end{array}\right) \cdot\left(\begin{array}{l}
x_{1} \\
x_{2}
\end{array}\right)+\left(\begin{array}{c}
0 \\
m_{23}
\end{array}\right) \cdot y\right] \cdot\left(x_{2}+y\right) \\
& Q_{0}(\underline{x}, y)=-(\gamma+\nu) y+\left[\left(m_{31}, m_{32}\right)\left(\begin{array}{l}
x_{1} \\
x_{2}
\end{array}\right)+m_{33} \cdot y\right] \cdot\left(x_{2}+y\right)
\end{aligned}
$$

and from order $\mathcal{O}(\mu)$

$$
\begin{aligned}
& \underline{P}_{1}(\underline{x}, y)=\left(\begin{array}{ll}
r_{11} & r_{12} \\
r_{21} & r_{22}
\end{array}\right)\left(\begin{array}{l}
x_{1} \\
x_{2}
\end{array}\right)+\left(\begin{array}{l}
r_{13} \\
r_{23}
\end{array}\right) y \\
& Q_{1}(\underline{x}, y)=\left(r_{31}, r_{32}\right)\left(\begin{array}{l}
x_{1} \\
x_{2}
\end{array}\right)+r_{33} \cdot y
\end{aligned}
$$

and the derivatives

$$
\begin{aligned}
\frac{\partial}{\partial y} \underline{P}_{0}(\underline{x}, y) & =\left(\begin{array}{c}
\xi \\
0
\end{array}\right)+\left(\begin{array}{cc}
m_{11} & 0 \\
-m_{31} & m_{22}+m_{23}
\end{array}\right) \cdot\left(\begin{array}{l}
x_{1} \\
x_{2}
\end{array}\right)+\left(\begin{array}{c}
0 \\
2 m_{23}
\end{array}\right) \cdot y \\
\frac{\partial}{\partial y} Q_{0}(\underline{x}, y) & =-(\gamma+\nu)+\left(m_{31}, m_{32}+m_{33}\right)\left(\begin{array}{l}
x_{1} \\
x_{2}
\end{array}\right)+2 m_{33} \cdot y
\end{aligned}
$$

we can now evaluate the $w_{1}(\underline{x})$-determining equation, Eq. (170), by inserting $y=h(\underline{x})=\mathcal{O}\left(\|\underline{x}\|^{2}\right)$, with

$$
h(\underline{x})=\underbrace{\left(x_{1}, x_{2}\right)\left(\begin{array}{cc}
0 & \frac{1}{2} h_{11} \\
\frac{1}{2} h_{11} & h_{02}
\end{array}\right)\left(\begin{array}{l}
x_{1} \\
x_{2}
\end{array}\right)}_{=\mathcal{O}\left(\|\underline{x}\|^{2}\right)}+\mathcal{O}\left(\|\underline{x}\|^{3}\right)
$$

and its derivative

$$
\frac{d h}{d \underline{x}}=\underbrace{\left(x_{1}, x_{2}\right)\left(\begin{array}{cc}
0 & h_{11} \\
h_{11} & 2 h_{02}
\end{array}\right)}_{=\mathcal{O}(\|\underline{x}\|)}+\mathcal{O}\left(\|\underline{x}\|^{2}\right),
$$

into all functions $\underline{P}_{0}, Q_{0}, \underline{P}_{1}, Q_{1}$ and $\frac{\partial}{\partial y} \underline{P}_{0}(\underline{x}, y), \frac{\partial}{\partial y} Q_{0}(\underline{x}, y)$ and determin the orders in $\underline{x}$ of the terms like

$$
\frac{d h}{d \underline{x}} \cdot \underline{P}_{1}=\underbrace{\left(x_{1}, x_{2}\right)\left(\begin{array}{cc}
0 & h_{11} \\
h_{11} & 2 h_{02}
\end{array}\right) \cdot\left(\begin{array}{ll}
r_{11} & r_{12} \\
r_{21} & r_{22}
\end{array}\right)\left(\begin{array}{l}
x_{1} \\
x_{2}
\end{array}\right)}_{=\mathcal{O}\left(\|\left.\underline{x}\right|^{2}\right)}+\mathcal{O}\left(\|\underline{x}\|^{3}\right)
$$

with the following results for the five terms involved in Eq. (170):

\section{E.3.1 Term $\frac{d h}{d \underline{x}} \cdot \underline{P}_{1}$}

From Eq. (188) we obtain the term $\frac{d h}{d \underline{x}} \cdot \underline{P}_{1}$

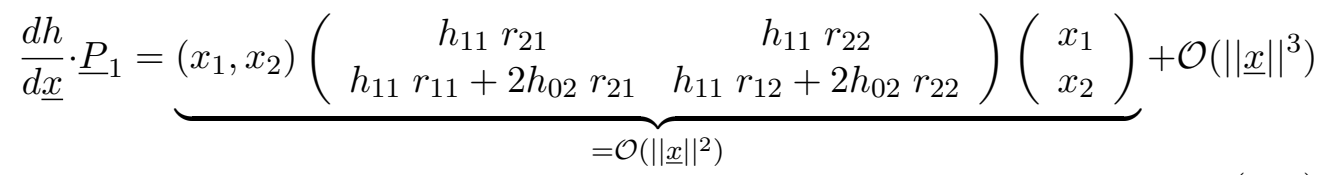


as one quadratic form plus higher order terms in $\underline{x}$. In this term no linear part is appearing.

\section{E.3.2 Term $\left.\frac{d h}{d \underline{x}} \cdot \frac{\partial}{\partial y} \underline{P}_{0}\right|_{y=h} \cdot w_{1}$}

For the second term in Eq. (170) we have

$$
\begin{aligned}
\left.\frac{d h}{d \underline{x}} \cdot \frac{\partial}{\partial y} \underline{P}_{0}\right|_{y=h} \cdot w_{1} & =\left(x_{1}, x_{2}\right)\left(\begin{array}{cc}
0 & h_{11} \\
h_{11} & 2 h_{02}
\end{array}\right) \cdot\left[\left(\begin{array}{c}
\xi \\
0
\end{array}\right)+\mathcal{O}(\|\underline{x}\|)\right] \cdot\left(w_{1,1}, w_{2,1}\right)\left(\begin{array}{c}
x_{1} \\
x_{2}
\end{array}\right)+\mathcal{O}(\|\underline{x}\|) \\
& =\underbrace{\left(x_{1}, x_{2}\right)\left(\begin{array}{c}
0 \\
\xi h_{11}
\end{array}\right) \cdot\left(w_{1,1}, w_{2,1}\right)\left(\begin{array}{l}
x_{1} \\
x_{2}
\end{array}\right)}_{=\mathcal{O}\left(\|\underline{x}\|^{2}\right)}+\mathcal{O}\left(\|\underline{x}\|^{3}\right)
\end{aligned}
$$

with the quadratic matrix

$$
\left(\begin{array}{c}
0 \\
\xi h_{11}
\end{array}\right) \cdot\left(w_{1,1}, w_{2,1}\right)=\left(\begin{array}{cc}
0 & 0 \\
\xi h_{11} w_{1,1} & \xi h_{11} w_{2,1}
\end{array}\right)
$$

for the quadratic form of the term $\left.\frac{d h}{d \underline{x}} \cdot \frac{\partial}{\partial y} \underline{P}_{0}\right|_{y=h} \cdot w_{1}$.

\section{E.3.3 Term $\frac{d w_{1}}{d \underline{x}} \underline{P}_{0}$}

For the third term we have

$$
\begin{aligned}
\frac{d w_{1}}{d \underline{x}} \underline{P}_{0}= & {\left[\left(w_{1,1}, w_{2,1}\right)+\left(x_{1}, x_{2}\right)\left(\begin{array}{cc}
2 w_{20,1} & w_{11,1} \\
w_{11,1} & 2 w_{02,1}
\end{array}\right)+\mathcal{O}\left(\|\underline{x}\|^{2}\right)\right] } \\
& \cdot\left[\left(\begin{array}{c}
\xi \\
0
\end{array}\right)\left(x_{2}+h(\underline{x})\right)+\left(\begin{array}{cc}
m_{11} & 0 \\
-m_{31} & m_{22}
\end{array}\right)\left(\begin{array}{l}
x_{1} \\
x_{2}
\end{array}\right) \cdot x_{2}+\mathcal{O}(\|\underline{x}\|)\right]
\end{aligned}
$$

and after some calculation, and baring in mind that only

$$
\left(w_{1,1}, w_{2,1}\right) \cdot\left(\begin{array}{cc}
m_{11} & 0 \\
-m_{31} & m_{22}
\end{array}\right)\left(\begin{array}{l}
x_{1} \\
x_{2}
\end{array}\right) \cdot x_{2}=\left(x_{1}, x_{2}\right)\left(\begin{array}{cc}
0 & \frac{1}{2}\left(w_{1,1} m_{11}-w_{2,1} m_{31}\right) \\
\frac{1}{2}\left(w_{1,1} m_{11}-w_{2,1} m_{31}\right) & w_{2,1}
\end{array}\right)\left(\begin{array}{l}
x_{1} \\
x_{2}
\end{array}\right)
$$

gives a standart quadratic form again, giving finally

$$
\begin{aligned}
\frac{d w_{1}}{d \underline{x}} \underline{P}_{0}= & \underbrace{\xi w_{1,1} x_{2}}_{=\mathcal{O}(\|\underline{x}\|)}+\underbrace{\left(x_{1}, x_{2}\right)\left[\left(\begin{array}{cc}
0 & \xi w_{1,1} \cdot \frac{1}{2} h_{11} \\
\xi w_{1,1} \cdot \frac{1}{2} h_{11} & \xi w_{1,1} \cdot h_{02}
\end{array}\right)\right.}_{=\mathcal{O}\left(\|\underline{x}\|^{2}\right)} \\
& \underbrace{\left.+\left(\begin{array}{cc}
\frac{1}{2}\left(w_{1,1}\right. & \left.m_{11}-w_{2,1} m_{31}\right) \\
w_{2,1}
\end{array}\right)+\left(\begin{array}{cc}
0 & \xi w_{20,1} \\
\frac{1}{2}\left(w_{1,1} m_{11}-w_{2,1} m_{31}\right) & \xi w_{11,1}
\end{array}\right)\right]\left(\begin{array}{l}
x_{1} \\
x_{2}
\end{array}\right)}_{=\mathcal{O}\left(\|\underline{x}\|^{2}\right)} \\
& +\mathcal{O}\left(\|\underline{x}\|^{3}\right)
\end{aligned}
$$

with two more terms of Eq. (170) to be evaluated. 


\section{E.3.4 Term $Q_{1}$}

The term

$$
Q_{1}(\underline{x}, y)=\left(r_{31}, r_{32}\right)\left(\begin{array}{l}
x_{1} \\
x_{2}
\end{array}\right)+r_{33} \cdot h(\underline{x})+\mathcal{O}\left(\|\underline{x}\|^{3}\right)
$$

giving

$$
\begin{aligned}
Q_{1}(\underline{x}, y)= & \underbrace{\left(r_{31}, r_{32}\right)\left(\begin{array}{l}
x_{1} \\
x_{2}
\end{array}\right)}_{=\mathcal{O}(\|\underline{x}\|)}+\underbrace{\left(x_{1}, x_{2}\right)\left(\begin{array}{cc}
0 & \frac{1}{2} h_{11} r_{33} \\
\frac{1}{2} h_{11} r_{33} & h_{02} \\
r_{33}
\end{array}\right)\left(\begin{array}{l}
x_{1} \\
x_{2}
\end{array}\right)}_{=\mathcal{O}\left(\|\underline{x}\|^{2}\right)} \\
& +\mathcal{O}\left(\|\underline{x}\|^{3}\right)
\end{aligned}
$$

has a linear part, not involving the coefficients $w_{1,1}$ and $w_{2,1}$, hence will finally give rise to non-vanishing linear coefficients in $w_{1}(\underline{x})$.

\section{E.3.5 Term $\left.\frac{\partial}{\partial y} Q_{0}\right|_{y=h} \cdot w_{1}$}

Finally, we evaluate the term

$$
\begin{aligned}
\left.\frac{\partial}{\partial y} Q_{0}\right|_{y=h} \cdot w_{1}= & {\left[-(\gamma+\nu)+\left(x_{1}, x_{2}\right)\left(\begin{array}{c}
m_{31} \\
m_{32}+m_{33}
\end{array}\right)+2 m_{33} \cdot h(\underline{x})\right] } \\
& \cdot\left[\left(w_{1,1}, w_{2,1}\right)\left(\begin{array}{c}
x_{1} \\
x_{2}
\end{array}\right)+\left(x_{1}, x_{2}\right)\left(\begin{array}{cc}
w_{20,1} & \frac{1}{2} w_{11,1} \\
\frac{1}{2} w_{11,1} & w_{02,1}
\end{array}\right)\left(\begin{array}{l}
x_{1} \\
x_{2}
\end{array}\right)\right]+\mathcal{O}\left(\|\underline{x}\|^{3}\right)
\end{aligned}
$$

giving

$$
\begin{aligned}
& \left.\frac{\partial}{\partial y} Q_{0}\right|_{y=h} \cdot w_{1}=\underbrace{-(\gamma+\nu) \cdot\left(w_{1,1}, w_{2,1}\right)\left(\begin{array}{l}
x_{1} \\
x_{2}
\end{array}\right)}_{=\mathcal{O}(\|\underline{x}\|)} \\
& \underbrace{\left(x_{1}, x_{2}\right)\left[-(\gamma+\nu) \cdot\left(\begin{array}{cc}
w_{20,1} & \frac{1}{2} w_{11,1} \\
\frac{1}{2} w_{11,1} & w_{02,1}
\end{array}\right)+\left(\begin{array}{c}
m_{31} \\
m_{32}+m_{33}
\end{array}\right)\left(w_{1,1}, w_{2,1}\right)\right]\left(\begin{array}{l}
x_{1} \\
x_{2}
\end{array}\right)}_{=\mathcal{O}\left(\|\underline{x}\|^{2}\right)} \\
& +\mathcal{O}\left(\|\underline{x}\|^{3}\right)
\end{aligned}
$$

so that now we have all terms of Eq. (170) and can hence calculate the coefficients of $w(\underline{x})$.

\section{E.3.6 Calculaton of the linear coefficients of $w_{1}$}

We now collect all linear terms in $\underline{x}$ in Eq. (170) to determin the linear coefficients of $w_{1}(\underline{x})$ as $w_{1,1}$ and $w_{2,1}$. We have only linear terms contributing from the terms $\frac{d w_{1}}{d \underline{x}} \underline{P}_{0}, Q_{0}$ and $\left.\frac{\partial}{\partial y} Q_{0}\right|_{y=h} \cdot w_{1}$, hence

$0=\left(0, \xi w_{11}\right)\left(\begin{array}{l}x_{1} \\ x_{2}\end{array}\right)-\left(r_{31}, r_{32}\right)\left(\begin{array}{l}x_{1} \\ x_{2}\end{array}\right)-\left(-(\gamma+\nu)\left(w_{1,1}, w_{2,1}\right)\left(\begin{array}{l}x_{1} \\ x_{2}\end{array}\right)\right)$ 
hence for the coefficients of $x_{1}$

$$
0-r_{31}+(\gamma+\nu) w_{1,1}=0
$$

giving

$$
w_{1,1}=\frac{r_{31}}{\gamma+\nu}
$$

and for the coefficients of $x_{2}$

$$
\xi w_{11}-r_{32}+(\gamma+\nu) w_{2,1}=0
$$

giving

$$
w_{2,1}=\frac{r_{32}-\xi w_{11}}{\gamma+\nu}
$$

\section{E.3.7 Calculaton of the quadratic coefficients of $w_{1}$}

Finally, we collect all quadratic terms in $\underline{x}$ in Eq. (170) to determin the quadratic coefficients of $w_{1}(\underline{x})$ as $w_{20,1}, w_{11,1}$ and $w_{02,1}$.

First we collect the quadratic terms depending on the quadratic coefficients $w_{i j, 1}$ and keep all other terms independent on $w_{i j, 1}$ in a matrix with elements $k_{i j}$ and obtain

$\left(x_{1}, x_{2}\right)\left[\xi\left(\begin{array}{cc}0 & w_{20,1} \\ w_{20,1} & w_{11,1}\end{array}\right)+(\gamma+\nu)\left(\begin{array}{cc}w_{20,1} & \frac{1}{2} w_{11,1} \\ \frac{1}{2} w_{11,1} & w_{02,1}\end{array}\right)\right]\left(\begin{array}{l}x_{1} \\ x_{2}\end{array}\right)=\left(x_{1}, x_{2}\right)\left(\begin{array}{cc}k_{11} & k_{12} \\ k_{21} & k_{22}\end{array}\right)\left(\begin{array}{l}x_{1} \\ x_{2}\end{array}\right)$

and labelling

$$
\left(\begin{array}{cc}
c_{11}\left(\left\{w_{i j, 1}\right\}\right) & c_{12}\left(\left\{w_{i j, 1}\right\}\right) \\
c_{21}\left(\left\{w_{i j, 1}\right\}\right) & c_{22}\left(\left\{w_{i j, 1}\right\}\right)
\end{array}\right):=\left[\xi\left(\begin{array}{cc}
0 & w_{20,1} \\
w_{20,1} & w_{11,1}
\end{array}\right)+(\gamma+\nu)\left(\begin{array}{cc}
w_{20,1} & \frac{1}{2} w_{11,1} \\
\frac{1}{2} w_{11,1} & w_{02,1}
\end{array}\right)\right]
$$

which gives the equation system for the coefficients $w_{i j, 1}$

$$
\left(x_{1}, x_{2}\right)\left(\begin{array}{ll}
c_{11}\left(\left\{w_{i j, 1}\right\}\right) & c_{12}\left(\left\{w_{i j, 1}\right\}\right) \\
c_{21}\left(\left\{w_{i j, 1}\right\}\right) & c_{22}\left(\left\{w_{i j, 1}\right\}\right)
\end{array}\right)\left(\begin{array}{l}
x_{1} \\
x_{2}
\end{array}\right)=\left(x_{1}, x_{2}\right)\left(\begin{array}{ll}
k_{11} & k_{12} \\
k_{21} & k_{22}
\end{array}\right)\left(\begin{array}{l}
x_{1} \\
x_{2}
\end{array}\right)
$$

and from which we obtain the coefficients of $x_{1}^{2}, x_{1} x_{2}$ and $x_{2}^{2}$ as three equations to determin $w_{20,1}, w_{11,1}$ and $w_{02,1}$ as follows. For the terms with powers $x_{1}^{2}$ we have the coefficients' equation given by

$$
c_{11}\left(w_{20,1}\right):=(\gamma+\nu) w_{20,1}=k_{11}
$$

hence the first coefficient is determined by

$$
w_{20,1}=\frac{k_{11}}{\gamma+\nu}
$$

ad further for $x_{1} x_{2}$ we have the coefficients' equation

$$
c_{12}\left(w_{11,1}, w_{20,1}\right)+c_{21}\left(w_{11,1}, w_{20,1}\right):=2 \xi w_{20,1}+(\gamma+\nu) w_{11,1}=k_{12}+k_{21}
$$


giving the second coefficient $w_{11,1}$ as function of the already known $w_{20,1}$ as

$$
w_{11,1}=\frac{1}{\gamma+\nu} \cdot\left(\left(k_{12}+k_{21}\right)-2 \xi w_{20,1}\right)
$$

And finally for $x_{2}^{2}$ we have the coefficients' equation

$$
c_{22}\left(w_{02,1}, w_{11,1}\right):=\xi w_{11,1}+(\gamma+\nu) w_{02,1}=k_{22}
$$

giving the final coefficient $w_{02,1}$ as function of the already known $w_{11,1}$ as

$$
w_{02,1}=\frac{1}{\gamma+\nu} \cdot\left(k_{22}-\xi w_{11,1}\right)
$$

with the $k_{i j}$ determined from Eq. (170) as

$$
\begin{aligned}
& \left(\begin{array}{ll}
k_{11} & k_{12} \\
k_{21} & k_{22}
\end{array}\right)=\left(\begin{array}{cc}
0 & \frac{1}{2} h_{11} \\
\frac{1}{2} h_{11} & h_{02}
\end{array}\right) \\
& +\left(\begin{array}{cc}
m_{31} w_{1,1} & m_{31} w_{2,1} \\
\left(m_{32}+m_{33}\right) w_{1,1} & \left(m_{32}+m_{33}\right) w_{2,1}
\end{array}\right) \\
& -\left(\begin{array}{cc}
h_{11} r_{21} & h_{11} r_{22} \\
h_{11} r_{11}+2 h_{02} r_{21} & h_{11} r_{12}+2 h_{02} r_{22}
\end{array}\right) \\
& -\left(\begin{array}{cc}
0 & 0 \\
\xi h_{11} w_{1,1} & \xi h_{11} w_{2,1}
\end{array}\right) \\
& -\xi w_{1,1} r_{33}\left(\begin{array}{cc}
0 & \frac{1}{2} h_{11} \\
\frac{1}{2} h_{11} & h_{02}
\end{array}\right) \\
& -\left(\begin{array}{cc}
0 & \frac{1}{2}\left(w_{1,1} m_{11}-w_{2,1} m_{31}\right) \\
\frac{1}{2}\left(w_{1,1} m_{11}-w_{2,1} m_{31}\right) & w_{2,1} m_{22}
\end{array}\right)
\end{aligned}
$$

with the results for $k_{i j}$

$$
\begin{aligned}
k_{11}= & m_{31} w_{1,1}-h_{11} r_{21} \\
k_{12}+k_{21}= & \left(1-\xi w_{1,1}\left(1+r_{33}\right)-\left(r_{11}+r_{22}\right)\right) h_{11} \\
& -2 h_{02} r_{21}+w_{1,1}\left(m_{32}+m_{33}-m_{11}\right)+2 w_{2,1} m_{31} \\
k_{22}= & \left(1-\xi w_{1,1} r_{33}-2 h_{02} r_{22}\right) h_{02}-h_{11} r_{12} \\
& +\left(m_{32}+m_{33}-m_{11}-\xi h_{11}\right) w_{2,1}
\end{aligned}
$$

so that we have completely determined the coefficients $w_{20,1}, w_{11,1}$ and $w_{02,1}$.

\section{E.4 Results for the coefficients of $w_{1}$}

We obtain for the linear part of $w_{1}(\underline{x})$

$$
\begin{aligned}
& w_{1,1}=\frac{r_{31}}{\gamma+\nu} \\
& w_{2,1}=\frac{r_{32}-\xi w_{1,1}}{\gamma+\nu}=\frac{r_{32}}{\gamma+\nu}-\xi \frac{r_{31}}{(\gamma+\nu)^{2}}
\end{aligned}
$$


and for the quadratic part

$$
\begin{aligned}
w_{20,1} & =\frac{k_{11}}{\gamma+\nu} \\
w_{11,1} & =\frac{1}{\gamma+\nu}\left(\left(k_{12}+k_{21}\right)-2 \xi w_{20,1}\right) \\
w_{02,1} & =\frac{1}{\gamma+\nu}\left(k_{22}-\xi w_{11,1}\right)
\end{aligned}
$$

with the functions $k_{i j}$, which depend only on model parameter and the already known coefficients of $w(\underline{x})$ in linear order in $\underline{x}$, given as

$$
\begin{aligned}
k_{11}= & m_{31} w_{1,1}-h_{11} r_{21} \\
k_{12}+k_{21}= & \left(1-\xi w_{1,1}\left(1+r_{33}\right)-\left(r_{11}+r_{22}\right)\right) h_{11} \\
& -2 h_{02} r_{21}+w_{1,1}\left(m_{32}+m_{33}-m_{11}\right)+2 w_{2,1} m_{31} \\
k_{22}= & \left(1-\xi w_{1,1} r_{33}-2 h_{02} r_{22}\right) h_{02}-h_{11} r_{12} \\
& +\left(m_{32}+m_{33}-m_{11}-\xi h_{11}\right) w_{2,1}
\end{aligned}
$$

\section{E.5 Results for the quadratic approximation of the center man- ifold $z_{3}=z_{3}\left(z_{2}, z_{1}\right)$ in next to leading order in $\mu$}

In original coordinates $z_{3}=z_{3}\left(z_{2}, z_{1}\right)$ we now have in $\mathcal{O}(\mu)$ to determin

$$
z_{3}=x_{2}+y
$$

with $y=w(\underline{x}, \mu)=h(\underline{x})+\mu w(\underline{x})+\mathcal{O}\left(\mu^{2}\right)$. Hence in quadratic order we have

$$
\begin{aligned}
y= & h_{02} x_{2}^{2}+h_{11} x_{1} x_{2}+\mathcal{O}\left(\|\underline{x}\|^{3}\right) \\
& +\mu\left(w_{1,1} x_{1}+w_{2,1} x_{2}+w_{20,1} x_{1}^{2}+w_{11,1} x_{1} x_{2}+w_{02,1} x_{2}^{2}+\mathcal{O}\left(\|\underline{x}\|^{3}\right)\right) \\
& +\mathcal{O}\left(\mu^{2}\right)
\end{aligned}
$$

and hence for $z_{3}$ we have

$$
\begin{aligned}
z_{3}= & \left(h_{02}+\mu w_{02,1}\right) \cdot x_{2}^{2} \\
& +\left(1+\mu w_{2,1}+\left(h_{11}+\mu w w_{11,1}\right) \cdot x_{1}\right) \cdot x_{2} \\
& +\mu\left(w_{1,1} x_{1}+w_{20,1} x_{1}^{2}\right)
\end{aligned}
$$

with $x_{1}=z_{1}$ and $x_{2}=\frac{1}{\varphi-\psi}\left(z_{2}-\psi z_{3}\right)$ and with further calculations analogously to the zeroth order calculation of the quadratic approximation described in the previous section. From this we obtain the quadratic equation in $z_{3}$ as

$$
z_{3}^{2}+p\left(z_{2}, z_{1}\right) \cdot z_{3}+q\left(z_{2}, z_{1}\right)=0
$$

with $p\left(z_{2}, z_{1}\right)$ and $q\left(z_{2}, z_{1}\right)$ given by

$$
p\left(z_{2}, z_{1}\right)=-\left(\frac{2}{\psi} z_{2}+\left(\frac{\varphi-\psi}{\psi}\right)^{2} \cdot \frac{1}{h_{02}+\mu w_{02,1}}\left(1+\frac{\psi}{\varphi-\psi}\left(1+\mu w_{2,1}+\left(h_{11}+\mu w_{11,1}\right) \cdot z_{1}\right)\right)\right)
$$


and

$q\left(z_{2}, z_{1}\right)=\frac{1}{\psi^{2}} z_{2}^{2}+\frac{\varphi-\psi}{\psi^{2}} \cdot \frac{1}{h_{02}+\mu w_{02,1}}\left(1+\mu w_{2,1}+\left(h_{11}+\mu w_{11,1}\right) \cdot z_{1}\right) \cdot z_{2}+\mu\left(w_{1,1} z_{1}+w_{20,1} z_{1}^{2}\right)$

and the solution

$$
z_{3}=-\frac{p}{2}+\sqrt{\left(\frac{p}{2}\right)^{2}-q} \approx \frac{1}{\varphi} \cdot z_{2}=\frac{\vartheta}{\nu} \frac{M}{N} \cdot z_{2}
$$

with $\mu$ dependent terms vanishing for $\mu$ going to zero, as can be seen from Eqs. (225) and (226), where $\mu$-dependent terms only appear additively next to zeroth order terms. 